\title{
OS TRATADOS DE GEORGE SALMON (1819 - 1904) NO CONTEXTO DA MATEMÁTICA BRITÂNICA NO SÉCULO XIX: DE UMA ABORDAGEM SINTÉTICA PARA UMA ABORDAGEM ANALÍTICA
}

\author{
Rodolpho Sousa Lima \\ Mestre PEMAT-UFRJ, Brasil \\ Gérard Emile Grimberg \\ Professor PEMAT-UFRJ, Brasil
}

(aceito para publicação em fevereiro de 2019)

\begin{abstract}
Resumo
O século XIX é um período divisor de águas na história da humanidade por apresentar os efeitos que a Revolução Industrial provocou na sociedade em geral, onde a Inglaterra foi um dos locais propulsores daquela época. Imersa nesse cenário de transformações, a matemática britânica deste período não ficou atrás em passar também por um processo de mudança. Neste sentido, destaca-se que a abordagem que se fazia presente naquele momento era herdada da tradição de Newton, Taylor e MacLaurin, a qual era fundamentada pela geometria pura ou também conhecida como geometrica sintética. Consciente de que a matemática britânica estava atrasada em relação à matemática dos demais países da Europa, um grupo de matemáticos britânicos formou a Analytical Society para introduzir uma nova abordagem fundamentada na geometria analítica e no cálculo diferencial leibniziano, desenvolvido no século anterior por matemáticos do continente, como Euler, d'Alembert e Lagrange. Esta nova perspectiva, então, proporcionou um avanço na matemática britânica. Essa transição de tratamento se manifesta nos artigos e livros de Peacock, Boole, Sylvester e Cayley, entre outros. George Salmon foi um dos matemáticos a expor essa nova abordagem em tratados didáticos. Se no primeiro tratado de Salmon, percebe-se um viés fortemente sintético, enquanto nos outros tratados posteriores, já se percebe uma abordagem analítica. Destacam-se nos tratados de Salmon os novos resultados dos geômetras do continente, além dos resultados da geometria projetiva que estavam esquecidos por mais de um século.
\end{abstract}

Palavras-chave: Matemática Britânica. George Salmon. Geometria Projetiva. Métodos Projetivos. Coordenadas Homogêneas. 


\title{
THE TREATIES OF GEORGE SALMON (1819 - 1904) IN THE CONTEXT OF BRITISH MATHEMATICS IN THE NINETEENTH CENTURY: A SYNTHETIC APPROACH TO AN ANALYTICAL APPROACH
}

\begin{abstract}
The nineteenth century is a watershed period in the history of mankind for presenting the effects of the Industrial Revolution on society in general, where England was one of the driving forces of that era. Immersed in this scenario of transformations, the British mathematics of this period was not far behind also undergoing a process of change. In this sense, it is emphasized that the approach that was present at that moment was that inherited from the tradition of Newton, Taylor and MacLaurin, which was based on pure geometry or also known as synthetic geometry. Aware of the fact that British mathematics was lagging behind the mathematics of other European countries, a group of British mathematicians formed the Analytical Society to introduce a new approach based on Leibniz's analytical geometry and differential calculus developed in the previous century by continent mathematicians, as Euler, d'Alembert and Lagrange. This new perspective, then, provided a breakthrough in British mathematics. This transition of treatment manifests itself in the articles and books of Peacock, Boole, Sylvester and Cayley, among others. George Salmon was one of the mathematicians to expose this new approach in didactic treatises. If in the first Salmon treatise one sees a strongly synthetic bias, whereas in the later treatises an analytic approach is already perceived. The Salmon treaties highlight the new results of the continent's geometers, as well as the results of Projective Geometry that had been forgotten for more than a century.
\end{abstract}

Keywords: British Mathematics. George Salmon. Projective Geometry. Projective Methods. Homogeneous Coordinates.

\section{Introdução}

Para entender as transformações que acontecem em um período é necessário conhecer qual é o panorama do momento para poder identificar os fatores que influenciam a ocorrência de mudanças. O período deste artigo abrange boa parte do século XIX. O século XIX representa uma ruptura para a matemática britânica, tanto para álgebra quanto para a geometria.

Este artigo procura encadear os fatos que transformaram a matemática britânica daquela época, a qual inicialmente era fundamentada numa abordagem sintética e, depois passa a ser influenciada pelos resultados analíticos que já se encontravam na matemática dos demais países da Europa.

A abordagem sintética na geometria era uma tendência herdada da tradição de Newton, Taylor e MacLaurin, e a matemática britânica se encontrava em um estado de atraso e de isolamento em relação à matemática do continente (DIAS, GRIMBERG, 2015). 
Um dos efeitos dessas transformações na geometria do século XIX, foi o desenvolvimento da geometria projetiva que, na época, era denominada de geometria descritiva.

A partir dos anos da década 1840, os artigos de Boole, Cayley e Sylvester participaram do desenvolvimento da geometria projetiva através da teoria dos invariantes. Um pouco depois, Salmon começou também a publicar artigos nesta área, bem como redigiu quatro tratados ${ }^{1}$ editados entre 1848 e 1862 (GOW, 1997).

A importância de Salmon não se dá apenas pelos tratados que ele escreveu, mas também por se tornar uma referência entre os matemáticos que utilizaram sucessivamente a abordagem sintética e a abordagem analítica. Suas duas primeiras obras refletem exatamente essa mudança de abordagem.

Apresentamos brevemente a metodologia na parte 2 desse artigo, o contexto da matemática britânica no período considerado na parte 3 , a convivência de dois pontos de visto, sintético e analítico, na geometria europeia da primeira metade do século XIX na parte 4, as partes 5, 6 e 7 apresentam a nossa análise dos trabalhos de Salmon, em particular, os conteúdos dos dois primeiros tratados. Enfim, seguem na parte 8, algumas considerações finais.

\section{Metodologia}

Para o desenvolvimento do nosso artigo, foram realizadas amplas leituras de artigos, de livros e de periódicos relacionados à matemática britânica do século XIX, dos métodos projetivos da geometria "moderna" daquele momento, e, sobretudo, dos tratados de George Salmon.

Apoiou-se, então, em estudos como: Projective Geometry and Mathematical Progress in Mid-Victorian Britain, de 1986, de Joan Richards, que analisa o desenvolvimento geral da matemática britânica, e trata também da evolução da geometria projetiva no período da nossa análise. Ainda da mesma autora, o artigo God, Truth, and Mathematics in Nineteeth Century England, de 1992, o qual traz também referências sobre esse período. Reforçando o cenário britânico nesse contexto, M. Fisch com 'The Emergency Which Has Arrived': the problematic History of Nineteenth-Century British Algebra, de 1994, que estuda o período de estagnação da matemática britânica decorrente ainda da forte tradição dos tratamentos sintéticos, e o movimento analítico liderado por Robert Woodhouse em busca de confrontar com a matemática de outros países do continente europeu que já estava bem mais avançada.

Além dos trabalhos de Joan Richards, utilizamos vários artigos e livros que embasaram a descrever as circunstâncias do desenvolvimento da matemática britânica: (CRILLY, 1986), (PARSHALL, 1989), (GOW, 1997), (LORENAT, 2015), (DIAS, GRIMBERG, 2015), (MENGHINI, 2019).

\footnotetext{
${ }^{1}$ Os títulos originais dos quatro tratados de George Salmon são: A treatise on Conic Sections (1848), A treatise on the higher plane curves (1852), Lessons introductory to the modern higher algebra (1859) e A treatise on the analytic geometry of three dimensions (1862).
}

RBHM, Vol. 19, n 38, p. 45-81, 2019 
Utilizamos as edições de 1850 e de 1855 de A Treatise on Conic Section, e as edições de 1852 e de 1879 de A treatise on the higher plane curves para efetuar a análise dos tratados escritos por George Salmon e realizar a comparação entre os tratamentos dados nas duas primeiras obras.

\section{O cenário da matemática britânica no século XIX}

O século XIX registrou os desdobramentos da segunda grande revolução na história da humanidade, que foi a Revolução Industrial, a qual já dava seus primeiros passos desde os meados do século anterior, promovendo uma nova estrutura da sociedade. Tal revolução se propagou tanto pela Europa quanto pela América, dando nascimento a chamada sociedade moderna, promovendo significativas mudanças no que diz respeito ao progresso tecnológico, desencadeando rapidamente uma era de investigações científicas sem precedentes, de modo que a profissionalização da ciência, em particular, da matemática, passou a ser reconhecida na Grã-Bretanha (RICHARDS, 1986, p. 297).

Presenciando todas as novidades que emergiam nos resultados da matemática, resultados aparentemente inconcebíveis começaram a ser desenvolvidos no século XIX. Na álgebra, por exemplo, desenvolveram-se álgebras diferentes daquela que havia até então. Hamilton, Grassmann e Cayley abriram os caminhos para a chamada álgebra abstrata.

Enquanto na geometria, a partir da negação do quinto postulado de Euclides, descobriu-se a existência de geometrias, diferentes da geometria tradicional, as chamadas geometrias não euclidianas (ROSENFELD, 1988).

Imersa nesse cenário, a matemática britânica naquele momento era vista como atrasada em relação à matemática do continente, pois ainda estava firmada na tradição da geometria euclidiana herdada de Newton. No decorrer do século XIX, a matemática britânica sofreu um processo de transformação na qual a visão sintética foi substituída por uma visão analítica.

\subsection{A primeira metade do século XIX}

O início do século XIX da matemática britânica foi marcado por importantes fatos que influenciaram na forma dos matemáticos desenvolverem suas pesquisas. Advinda de uma tradição herdada dos trabalhos de MacLaurin, Newton e Taylor, tal matemática estava fortemente influenciada no uso exclusivo da geometria sintética que se apoiava na geometria de Arquimedes e de Euclides, usada para demonstrar todas as propriedades das curvas, fazendo com que a matemática britânica criasse uma resistência a todo desenvolvimento da análise e do cálculo diferencial, colocando-se numa situação de isolamento (DIAS, GRIMBERG, 2015, p.15).

Ball (1889) explica o isolamento pela diferença de práticas:

“O fato principal é a questão dos matemáticos britânicos não realizarem um esforço semelhante, isto é, de usar também as notações e os resultados da matemática do continente, 
colocando-se, dessa forma, em situação de distanciamento em relação aos seus vizinhos" (BALL, 1989, pp. 97-98). ${ }^{2}$

Sobre esse fato se percebe que a escola newtoniana insistia em usar demonstrações geométricas, mesmo após os princípios do cálculo diferencial leibniziano já se terem tornados universais. Richards (1986, p. 306) também evidencia outro motivo desta visão sintética: o caráter tradicionalista e conformista da educação britânica.

Porém, no início do século XIX, uma perspectiva nova foi aberta por Robert Woodhouse $^{3}$, que foi o primeiro a publicar um trabalho ${ }^{4}$ que introduzia os conceitos do cálculo diferencial leibniziano. Este matemático explicou que os matemáticos ingleses deveriam converter-se às notações do cálculo diferencial de Leibniz e reuniu um grupo de matemáticos interessados em introduzir as inovações analíticas do cálculo diferencial do século XVIII que permeavam a pesquisa matemática europeia.

Este grupo formado por matemáticos seguidores das ideias de Woodhouse constituiu assim a chamada Analitical Society, a qual, além de procurar romper a resistência aos novos tratamentos analíticos, procurou também retirar a matemática britânica do seu estado de isolamento e de atraso em relação à matemática do continente europeu.

A questão da escolha da notação de Newton usada na matemática britânica em oposição à notação de Leibniz usada pelos demais países da Europa não é suficiente para explicar o atraso britânico, pois mesmo que a notação de Newton fosse considerada superior à notação de Leibniz (o que não é o caso), os matemáticos britânicos ainda assim estariam em desvantagem em relação ao restante da comunidade matemática europeia por ainda utilizar quase unicamente os resultados de Newton,Taylor de Maclaurin, (DIAS, GRIMBERG, 2015, p. 9).

Além do ganho obtido com as novas notações do cálculo diferencial, houve também uma abertura para pesquisas sob o ponto de vista analítico, com destaque para a álgebra com aplicações geométricas. Destaca-se ainda a criação de um periódico inglês específico para publicações de artigos matemáticos, The Cambridge Mathematical Journal, onde se pode encontrar a presença de diversos trabalhos relacionados à geometria analítica e a álgebra aplicada à geometria, dando a clara noção das mudanças que estavam ocorrendo nos anos iniciais da primeira metade do século XIX na matemática britânica.

Em Fisch (1994), encontra-se uma contextualização desse período, indicando, de fato, as mudanças que estavam ocorrendo na matemática britânica naquele momento:

\footnotetext{
${ }^{2}$ A common language and facility of intercommunication of ideas are of the most importance in science, and even if the Cambridge school had enjoyed the use of a better notation than their continental contemporaries they would have lost a great deal by their isolation. So little however did they realize this truth that they made no serious efforts toke ep themselves acquainted with the development of analysis by their neighbors.

${ }^{3}$ Robert Woodhouse $(1773$ - 1827) professor de matemática em Cambridge, em 1820 foi eleito Lucasion Professor, cadeira de matemática de grande prestígio que foi ocupada por Isaac Newton.

${ }^{4} \mathrm{O}$ título deste trabalho é Principles of analytical calculation que foi publicado em $1803 \mathrm{em}$ Cambridge.
}

RBHM, Vol. 19, n 38, p. 45-81, 2019 
"A notável revitalização da matemática britânica e da física matemática durante a primeira metade do século XIX é talvez a mais merecedora de destaque" (FISCH, 1994, p. 247). ${ }^{5}$

Fisch comenta que o processo de reforma foi sendo efetivamente implementado pela Cambridge Analytical Society ${ }^{6}$ no início dos anos de 1810 . Embora não tenha sido a primeira a fazer críticas ao estado que se encontrava a matemática britânica na época, de certa forma, esta sociedade promoveu uma reforma que proporcionou os vários efeitos na matemática britânica.

As duas primeiras conquistas marcantes dessa Sociedade que mereceram destaques foram: em 1816, a publicação da tradução do livro-texto Elementary differential calculus, de Lacroix, e, em 1817, a introdução por Peacock dos símbolos do cálculo diferencial no

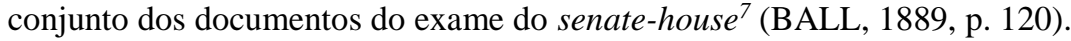

A reforma proposta pela Analytical Society [Sociedade Analítica] foi de suma importância para colocar a matemática britânica em novos rumos e deve-se efetivamente ao mais influente dos membros dessa sociedade, George Peacock, a responsabilidade por retirar os matemáticos britânicos do isolamento em relação ao restante da Europa, evidenciando mais uma vez esse cenário que já perdurava desde a última metade do século XVIII.

Porém, não se pode deixar de considerar os trabalhos anteriores, como o de Robert Woodhouse, em Cambridge, bem como destacar a existência de muitos outros centros de reforma tão importantes quanto da Analytical Society, dentre os quais, podemos destacar as academias militares ${ }^{8}$, até as universidades, como Oxford, Edimburgo, Glasgow e Dublin, demonstrando que a matemática britânica como um todo, realmente, estava fundamentada nos princípios sintéticos e, que a partir desses movimentos, começou a receber essa influência analítica (GUICCIARDINI, 1989, p. 136).

Logo, conforme a escola analítica ia vencendo as barreiras impostas pela tradição de Newton, não houve somente um ganho nos métodos analíticos e do cálculo diferencial, mas também ocorreu uma abertura para a matemática continental, inclusive para esses métodos de aplicações da álgebra para geometria (DIAS, GRIMBERG, 2015, p. 13).

Ainda na primeira metade do século XIX, a partir dos desenvolvimentos de Gauss e Lagrange em substituições lineares, destaca-se o surgimento de pesquisadores ingleses, como Augustus de Morgan, interessados em desenvolver as propriedades dessas substituições e de suas aplicações à geometria e, consequentemente, às demais áreas das ciências físicas, como a mecânica.

\footnotetext{
${ }^{5}$ The remarkable revitalization of British mathematics and mathematical physics during the first half of the nineteenth century is perhaps them most deserving of the name.

${ }^{6}$ Cambridge AnalyticalSociety criada em 1812 pelos matemáticos George Peacock (1791 - 1858), John Herschel (1792 - 1871) e Charles Babbage (1791 - 1871). Seguindo as influências das observações de Woodhouse, os três matemáticos concordaram em formar esta Sociedade com o objetivo de defender o uso dos métodos analíticos e da nova notação do cálculo diferencial no lugar da antiga notação de Newton.

${ }^{7}$ Senate-house é o centro administrativo da Universidade de Londres.

${ }^{8}$ Pode ser citada, por exemplo, a Academia Royal Military Academy at Woolwich.
} 
Richards (1986, p. 308) reforça essa constatação ao comentar que podiam ser encontradas publicações em artigos nos jornais matemáticos, onde as tentativas de fornecer interpretações geométricas para resultados analíticos aumentaram substancialmente nessa década e, cada vez mais, a importância em fundamentar os resultados analíticos foi ganhando espaço. Com isso, percebe-se que os métodos analíticos e sintéticos se permeavam e intensificaram-se a partir da década de 1840.

\subsection{A segunda metade do século XIX}

Ao entrar na segunda metade do século XIX, a busca pelo desenvolvimento científico se intensificou e tornou-se uma profissão reconhecida na Grã-Bretanha, de modo que esse reconhecimento favoreceu que os profissionais de área começaram a receber incentivos educacionais e institucionais. Assim muitos cientistas britânicos, em particular os matemáticos, engajaram-se ativamente para promover esse desenvolvimento como, por exemplo, os novos resultados a respeito dos métodos projetivos que ocorreram nesse momento (RICHARDS, 1986, p. 297).

Esse engajamento já se notava desde o início da década anterior, ao identificar o quanto as produções na matemática britânica, em especial, as pesquisas em geometria analítica se tornaram expressivas. Tal fato se percebe ao fazer uma breve leitura no indíce do segundo volume do The Cambridge Mathematical Journal de 1841, que, além de confirmarem a alta produção de publicações da matemática britânica, também apontam como os desenvolvimentos analíticos evoluíram de modo que uma seção ${ }^{9}$ específica nesse periódico foi criada para divulgar esses resultados. Com relação à parte destinada à álgebra do referido jornal, não foi difícil encontrar trabalhos relacionando a álgebra à geometria, como o artigo de Boole, Researches in the theory of analytical transformations, with a special application to the reduction of the general equation of the second order, publicado em 1841 (PARSHALL, 1989, p. 160).

A importância dessas publicações a partir da década de 40 é devida à forte influência que Boole exerceu sobre os trabalhos de Cayley ${ }^{10}$ e Sylvester ${ }^{11}$, principalmente ao introduzir e definir os conceitos da teoria dos invariantes ${ }^{12}$ (ibid., p. 162). Os trabalhos de Salmon se elaboram nesse contexto e serão analisados nas partes 5, 6 e 7 do artigo.

Nesse período, Cayley criou uma relação de amizade com Sylvester, com quem fez grande parceria no desenvolvimento da teoria dos invariantes. Por volta de 1850, Salmon deu algumas contribuições a essa teoria.

\footnotetext{
${ }^{9} \mathrm{O}$ título desta seção específica é Analytical geometry of three dimensions.

${ }^{10}$ Arthur Cayley $(1821$ - 1895) foi um dos mais produtivos matemáticos britânicos da sua época, sendo um dos atuantes nos desenvolvimentos das Teorias dos Invariantes, das Matrizes e dos Determinantes.

11 James Joseph Sylvester (114 - 1987) matemático britânico que contribuiu fundamentalmente no desenvolvimento da teoria matricial, da teoria dos invariantes, teoria dos números e análise combinatória. Desempenhou papel fundamental no desenvolvimento da matemática nos Estados Unidos na segunda metade do século XIX, quando professor da Universidade Johns Hopkins e fundador do American Journal of Mathematics.

${ }^{12}$ Teoria dos Invariantes é, essencialmente, funções dos coeficientes que são preservadas por mudanças de coordenadas.
} 
Richards (1986) destaca que essa "nova" geometria - a geometria projetiva - estava servindo de interpretação para novos resultados analíticos e que a paixão britânica pela álgebra dos invariantes foi ganhando força no meado do século:

"A partir de um artigo" de George Boole e de matemáticos como Arthur Cayley, J. J. Sylvester e o irlandês George Salmon, estabeleceram a tarefa de descobrir as propriedades das expressões homogêneas, dos invariantes de uma transformação linear" (RICHARDS, 1986, p. 308) ${ }^{14}$.

Richards comenta ainda que o estudo dos polinômios homogêneos, em particular das formas quadráticas, tornou-se um domínio de estudo importante que motivou a publicação desses novos conceitos em livros, como os escritos por George Salmon, defendendo os métodos analíticos que eram percebidos como sendo parte integrante dos avanços realizados por Möbius e Plücker.

Com o desenvolvimento das propriedades das expressões algébricas, dos invariantes de uma transformação linear, outro exemplo significativo desse movimento na matemática foi a utilização das coordenadas homogêneas para o estudo das curvas algébricas, o que situava de fato o domínio dessas curvas no plano projetivo.

A projeção do desenvolvimento dessas teorias também influenciou nos periódicos, como podemos destacar a expansão do jornal The Cambridge Mathematical Journal para o The Cambridge and Dublin Mathematical Journal, a partir de 1846 e também quando Cayley iniciou a divulgação dos seus resultados na Europa, com a publicação do artigo Mémoire sur les Courbes à double courbure, no jornal de Liouville ${ }^{15}$, que era um dos mais importantes periódicos internacionais, juntamente com o jornal de Crelle $^{16}$ (CAYLEY, 1845a).

A expansão da revista The Cambridge Mathematical Journal não tinha só a pretensão de ter um caráter de continuidade, mas também sinalizava promover uma maior abertura a trabalhos internacionais, seguindo os planos da Analytical Society de atualizar e integrar a matemática britânica. Para exemplificar essas publicações promovidas por essa abertura, podemos encontrar já no primeiro volume um artigo de Liouville, no terceiro volume, um artigo de Charles Hermite, ambos matemáticos franceses. No oitavo volume e no nono aparecem artigos de Hermite, Steiner e Brioschi.

O uso da álgebra aplicada à geometria não era só a prática comum a Cayley, Sylvester e Salmon, mas também a outros contemporâneos britânicos, e que a partir de 1850 , deram novos rumos à teoria dos invariantes.

${ }^{13} \mathrm{O}$ artigo do qual Richards se refere é 'Exposition of a General Theory of Linear Transformations, Parts I and II', Cambridge Mathematics Journal 3 (1841), 1-20, 106-119.

${ }_{14}$ "Taking off from an initial paper by George Boole, men like Arthur Cayley, J. J. Sylvester and the Irishman George Salmon, set themselves the task of discovering those properties of homogeneous expressions which are invariant under linear transformations."

${ }^{15} \mathrm{O}$ Journal de mathématiques pures et appliquées (JMPA) é uma publicação científica francesa, fundado em 1836 pelo matemático Joseph Liouville, sendo por isto também conhecido como Journal de Liouville.

${ }^{16}$ O Journal de Crelle é o nome comum dado ao periódico matemático alemão Journal für die reine und angewandte Mathematik, fundado em 1826 em Berlin, pelo matemático August Leopold Crelle. 
Nesse período, Boole também utilizou substituições lineares para o estudo das curvas algébricas. O índice do quarto volume do The Cambridge Mathematical Journal, de 1845, aponta esse fato mostrando o quanto os métodos analíticos aplicados à geometria avançaram, uma vez que a seção destinada à geometria plana já apresentava vários artigos que seguiam esta tendência.

Por sua vez, a geometria considerada foi a geometria das propriedades projetivas e de suas transformações, a qual chega a Cambridge por intermédio da influência francesa, de Chasles, e da influência alemã, a partir dos textos de Plücker e Hesse.

Aliás, Cayley tinha publicado mais artigos nos jornais internacionais, como os jornais de Crelle e de Liouville, do que no The Cambridge and Dublin Mathematical Journal, na década 1840, artigos estes que tratavam sobre a "teoria das curvas", na perspectiva de Plücker, no uso dos polinômios homogêneos e das teorias de Eisenstein, e de Hesse.

Esses destaques às teorias de Hesse, Eisenstein, e Chasles apontam como a abertura da matemática britânica aos conceitos desenvolvidos pela matemática do continente, em particular, da matemática alemã e francesa, acabou com o isolamento que se via no início do século XIX, bem como a distância da comunicação que se encurtou através das publicações nos periódicos The Cambridge and Dublin Mathematical Journal, Journal de Crelle e Journal de Liouville.

Esses artigos mostram que além do uso das coordenadas homogêneas já serem uma prática bem estabelecida, a retomada ao uso das coordenadas cartesianas também era uma prática em que se demonstrava total domínio. Tais práticas podem ser observadas nos resultados publicados no segundo tratado de Salmon.

Tais influências são registradas nas publicações de George Salmon, sobretudo na sua segunda obra que iremos analisar e deve-se principalmente ao trabalho conjunto com Cayley, o qual em seus artigos publicados no periódico de Liouville traz não só referências à "Teoria das curvas algébricas de M. Plücker", como também se propõe aplicar os princípios desta teoria às curvas no espaço de três dimensões, esclarecendo as possíveis relações entre pontos, curvas e planos. (CAYLEY, 1845, p. 245).

Em outro artigo de Cayley, cujo título é Démonstration d'un Théorème de M. Chasles, publicado no jornal de Liouville, pode-se perceber que além de confirmar a influência francesa através da teoria geométrica de Chasles, destaca-se também a presença do uso das coordenadas homogêneas, dando algumas características do uso da álgebra das substituições lineares aplicada à geometria projetiva (CAYLEY, 1845b).

Essa prática comum entre os matemáticos mostra o relacionamento que havia entre eles ao se comunicarem através de seus artigos. Como se pode perceber, Boole influenciou fortemente os trabalhos de Cayley e este, consequentemente, os trabalhos de Sylvester e de Salmon (CRILLY, 1986). No artigo de Cayley, On geometrical reciprocity, de 1848, observa-se que Cayley cita Plücker como fonte de inspiração em seus trabalhos com as cônicas e as coordenadas homogêneas, em particular para o "teorema fundamental da reciprocidade", o qual se refere à dualidade no plano, estudando as propriedades das cônicas, cada uma delas com o seu respectivo teorema dual.

Destacam-se também os trabalhos realizados por Sylvester, o qual contribuiu com diversos artigos no início da segunda metade do século XIX relacionados às propriedades 
projetivas das cônicas, como On the intersections, contacts, and other correlations of two conics expressed by indeterminate coordinates que estuda propriedades de superfícies, usando coordenadas do espaço projetivo (SYLVESTER, 1850).

Em seu artigo de 1851, cujo título é On the general theory of associated algebraical forms, Sylvester apresentou uma nova nomenclatura dos principais componentes da teoria dos invariantes e foi nesse artigo que a teoria recebe esse nome, conforme ele apresentou e utilizou em seu artigo (SYLVESTER, 1851).

O teor analítico dos artigos dos matemáticos britânicos no início da segunda metade do século XIX levou Salmon a escrever o seu segundo tratado.

Com efeito, Salmon participou do desenvolvimento da teoria dos invariantes utilizando também esses métodos analíticos, que após o seu primeiro trabalho cujo foco ainda era sintético, a partir do segundo tratado, passou a ser analítico. Salmon também se concentrou em trabalhos que utilizava os polinômios homogêneos, para estudo das superfícies e de suas propriedades projetivas, como pode ser visto em seus artigos, de 1851, Théorèmes sur les courbes de troisième degrée sur la formation de l'équation de la courbe reciproque à une courbe donnée e, de 1856, On the degree of a surface reciprocal to a given one.

Além disso, destacamos também a importância que os tratados de George Salmon ganharam ao se tornarem livros textos de referência na Inglaterra e na Irlanda e, consequentemente, nos demais países do continente europeu bem como na América.

$\mathrm{O}$ artigo On The Degree of a superface reciprocal to a given one de Salmon, que também trabalhou sobre o assunto das cônicas e sua relação algébrica (SALMON, 1846), é citado por Cayley em On the triple tangent planes of surfaces of the third order (CAYLEY, 1849, p. 446) o que mostra que, tanto Cayley quanto Salmon, ambos pesquisaram o método da polar recíproca, assunto o qual se encontra no primeiro tratado de Salmon e também destaca o caso no qual as superfícies são de segunda ordem. Salmon também considerou teoremas nas curvas de terceira ordem, assim como Cayley, e relacionou a geometria projetiva entre retas e cônicas.

Como tem sido apresentada até agora, a proposta comum entre os diversos textos é a metodologia dada ao tratamento da álgebra dos polinômios homogêneos com aplicações geométricas associadas às propriedades projetivas dessas curvas que estes polinômios representam. Daí o campo de pesquisa na matemática britância fica bem caracterizado por essa prática comum, característica na segunda metade do século XIX.

Apesar da clara utilização de aspectos geométricos, os diversos trabalhos não apresentam figuras, nem mesmo para exemplificar. Isso pode ser compreendido como uma mudança de postura a partir da Analytical Society, retirando a geometria sintética da posição de único padrão de rigor, mantendo-se, porém, uma visão geométrica que ajuda no raciocínio matemático das diversas teorias algébricas.

Aliás, a visão geométrica é mais do que uma ferramenta, pois, se de um lado influencia diretamente a interpretação dos resultados da teoria dos invariantes, de outro lado, irá revolucionar a relação da geometria projetiva com as geometrias euclidiana e não euclidianas, a partir da leitura dos artigos de Cayley sobre os Quantics por Félix Klein. 


\section{A geometria projetiva, da visão sintética à visão analítica}

O objetivo do capítulo será de apresentar o motivo pelo qual o desenvolvimento da geometria projetiva no século XIX despertou um grande interesse na comunidade matemática daquela época com os novos resultados que estavam sendo publicados. Richards (1986) dá uma amostra ao apresentar o tratamento que esses métodos projetivos receberam na época:

\section{"Desde o início, este estudo foi indistintamente denominado como "geometria descritiva", "geometria recente", ou "geometria moderna”” (RICHARDS, 1986, p. 301) ${ }^{17}$.}

A importância do desenvolvimento desses novos resultados da geometria projetiva poderá ser conferida no primeiro tratado de Salmon, onde ele apresenta os conceitos dessa geometria, bem como os resultados de Poncelet e Chasles, sobretudo no que diz respeito à perspectiva sintética nessa obra.

A visão preponderante que havia e que permaneceu, praticamente, até o final do século XVIII, era de que a geometria euclidiana fosse uma descrição fiel do mundo sensível, a qual era tratada como um dogma. Entretanto tal visão foi questionada e no decorrer do século XIX se desenvolveram várias geometrias, além da projetiva, a geometria de inversão, as geometrias não euclidianas, e a geometria diferencial.

Essa diversidade de teorias marcou uma libertação do mundo físico a partir da aceitação de objetos coerentemente concebidos pela mente humana.

A partir dessa aceitação, com relação à geometria projetiva, constata-se, por exemplo, o fato de que duas retas paralelas que na geometria de Euclides não possuía um ponto em comum, agora, são concorrentes em um ponto no infinito.

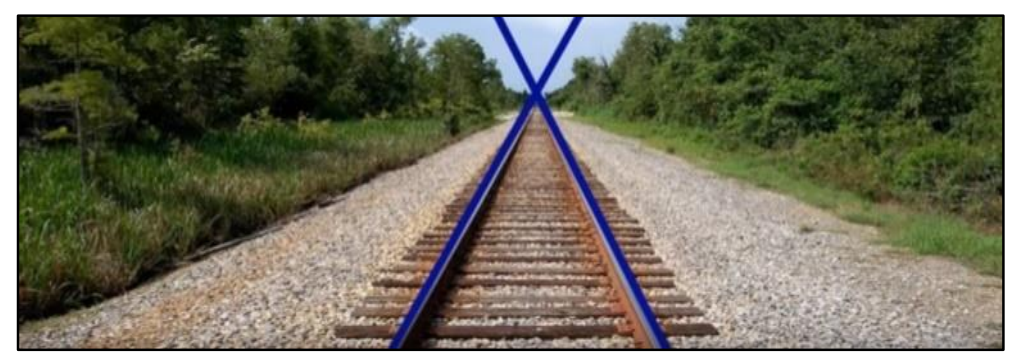

Figura 1: Ideia de duas retas paralelas se interceptando no infinito.

Devido a todas essas transformações que estavam ocorrendo na época, também se pode perceber as mudanças que aconteceram na forma como os resultados eram publicados, a metodologia sintética se fazia bem presente no início do século XIX nos resultados

\footnotetext{
${ }^{17}$ From its inception, this study, which was then rather indiscriminately designated as "descriptive geometry", "projective geometry", "recente geometry", or "modern geometry".
} 
também da geometria projetiva, e, com o passar do tempo, começou a dividir o espaço com a metodologia analítica, que já se evidenciava nos anos finais do século XVIII.

Embora inicialmente essas correntes convivessem bem no início do século XIX em decorrência do ensino de Monge iniciando resultados dessa "nova" geometria. Após sua morte, tornou-se evidente o confronto entre duas abordagens. A partir dessa rivalidade, analisa-se a passagem da visão sintética para visão analítica, assim como na evolução dos trabalhos de George Salmon por volta de 1850.

\subsection{O contexto histórico}

Datam da Antiguidade Clássica os primeiros resultados que hoje são considerados do âmbito da geometria projetiva. Trabalho como o de Apolônio de Perga, como o seu famoso tratado sobre seções cônicas, ou os Porismos de Euclides, que chegaram até nós através do comentário de Pappus, exemplificam essas primeiras evidências. No entanto, o seu desenvolvimento começou realmente no século XVI, em consequência dos trabalhos sobre a perspectiva no período do Renascimento.

Nesse período se registra o primeiro grande tratado publicado sobre cônicas desde a Antiguidade, o qual se deve a Johannes Werner, bem como a introdução por Johannes Kepler dos conceitos de pontos e retas no infinito para o estudo das cônicas.

Considera-se que um dos grandes passos dado para o desenvolvimento da geometria projetiva foi dado por Girard Desargues, o qual obteve vários resultados no estudo das cônicas sob o ponto de vista projetivo, introduzindo diversos conceitos fundamentais, como os de involução, polares e quadriláteros completos.

Entretanto os trabalhos de Desargues não obtiveram muito impacto na época, devido ao seu estilo de exposição difícil e pouco convencional, sobretudo pelo fato de seus métodos constituírem também uma ruptura tanto com a tradição clássica quanto com a geometria analítica que começava a despontar naquele momento. Dessa forma, os trabalhos de Desargues acabaram não tendo uma continuidade.

Blaise Pascal desenvolveu os métodos de Desargues culminando na edição do conhecido Essay pour les coniques, de 1640, onde se encontra a publicação do teorema do hexagrama místico e seus respectivos corolários.

Após Pascal, o desenvolvimento das pesquisas sobre propriedades da geometria projetiva novamente foi abandonado e, praticamente, substituído pela geometria analítica cartesiana que estava em alta naquele momento. Os poucos resultados que se pode encontrar devem-se a Phillipe de La Hire com o seu trabalho sobre cônicas tratadas projetivamente e, também, a alguns resultados obtidos por Braikenridge, Maclaurin e Newton no início do século XVIII. Tais resultados reaparecem apenas no início do século XIX.

O ressurgimento da geometria projetiva efetivamente se deve a um grupo de alunos da École Polytechnique ${ }^{18}$, o qual foi essencialmente formado por Charles Brianchon,

\footnotetext{
${ }^{18}$ École Polytechnique é uma das mais antigas e prestigiosas escolas de engenharia francesa, fundada em 1794 reconhecida pela proeminência da matemática na formação de seus alunos.
} 
que demonstrou o teorema dual do teorema de Pascal, Michel Chasles, Joseph Gergonne e, sobretudo, Jean Victor Poncelet, grande defensor dos métodos sintéticos em geometria. O lado analítico da geometria projetiva, por outro lado, deve seus progressos extraordinários aos trabalhos de Möbius, e, sobretudo, de Julius Plücker.

No fim do século XIX e no começo do XX a geometria projetiva recebeu muitos tratamentos axiomáticos e descobriram-se geometrias projetivas finitas. Com isso mostrouse que, com graduais acréscimos e alterações de postulados, pode-se passar da geometria projetiva para a geometria euclidiana, encontrando-se muitas outras geometrias no caminho.

Com relação ao desenvolvimento da estrutura dessa nova geometria, destacam-se os seguintes pontos: na geometria projetiva, não existe o conceito de retas paralelas, pois são adicionados no plano afim usual os pontos "no infinito", os quais foram introduzidos por Kepler, de modo que duas retas quaisquer se intersectam sempre num ponto. Nessa geometria também não fazem sentido conceitos métricos, não há tratamento com ângulos e com distâncias. Entretanto, há outros conceitos e propriedades que podem ser analisados, como as relações de incidência e de colinearidade entre objetos diferentes.

Com a introdução dos pontos no infinito, que também são chamados de pontos de fuga (na teoria da perspectiva), a geometria projetiva permitiu uniformizar as cônicas, em outras palavras, nessa geometria não se faz a distinção entre as cônicas: elipses, parábolas ou hipérboles, pois com a inclusão de uma determinada quantidade de pontos no infinito, as cônicas se transformam por projeção no mesmo tipo de cônica projetiva, desde que não forem degeneradas (CHASLES, 1837).

Assim, qualquer propriedade projetiva que se verifique para uma cônica projetiva não degenerada em particular, verifica-se também para qualquer outra. Logo, isso implica que as demonstrações das propriedades relativas às cônicas projetivas se tornam muito mais simples porque é possível particularizar essas demonstrações para uma cônica projetiva não degenerada de equação mais simples ou que passe em determinado conjunto de pontos até chegar à conclusão de que a propriedade é válida para qualquer outra cônica projetiva não degenerada.

Outro aspecto muito interessante na geometria projetiva é o chamado princípio da dualidade, princípio esse que permite que se permutem alguns termos nos enunciados das propriedades, como, por exemplo, ponto por reta, reta por ponto, ou colineares por concorrentes. Dessa forma, obtêm-se novas propriedades a partir de outras já demonstradas sem a necessidade de prová-las novamente.

\subsection{Da visão sintética}

O objetivo aqui será de procurar esclarecer o que trata a visão sintética da geometria, a qual era predominante nos resultados no início do século XIX e que depois passou a disputar a concorrência com os métodos analíticos.

Sendo resgatada e desenvolvida no final do século XVIII e avançando para o seguinte, primeiramente por Monge, cuja importância é considerada não só pelas suas próprias contribuições, mas, especialmente, porque ele abriu os caminhos em favor de um 
retorno à geometria sintética no século XIX e deixou esse legado para ser desenvolvido ao grupo de alunos da École.

A continuação do desenvolvimento dada à geometria "moderna" por Poncelet e Chasles representou, assim, a primeira grande extensão dos métodos sintéticos em oposição ao método analítico.

Poncelet procurou tornar seus enunciados de geometria sintética os mais gerais possíveis, contrapondo o que ele percebia em relação às vantagens que os metódos analíticos pareciam ter, que residia exatamente em suas generalidades. Um dos seus enunciados que fortalece esse posicionamento é o enunciado que ele chamou de princípio de continuidade ${ }^{19}$ (LORENAT, 2015, pp. 157-158).

Destaca-se um fato curioso sobre Poncelet: embora ele inicialmente tivesse escrito um tratado sobre geometria analítica entre 1813 e 1814, cujo título é Applications d'analyse et de géométrie, esse trabalho, porém, só foi publicado ${ }^{20}$ no meio do século XIX, bem depois da publicação do seu famoso tratado publicado em 1822, Traité des propriétés projectives des figures, a qual se destingue das outras exatamente pela seu caráter sintético.

Assim, Poncelet foi quem deu o impulso definitivo para o desenvolvimento da geometria projetiva. Ele foi o primeiro a considerar a geometria projetiva como um novo ramo da matemática com objetivos e métodos próprios, distinguindo entre propriedades que são projetivas e aquelas que não são (ibid., p. 156).

Em relação a Michel Chasles, embora na parte anterior, mencionou-se sua participação no lado analítico da geometria projetiva, o destaque feito a ele é devido ao mesmo ter sido também um grande especialista em geometria sintética. Tal fato pode ser visto no seu trabalho Aperçu Historique sur l'Origine et le Développement des Méthodes em Géométrie de 1837.

Essa perspectiva também foi seguida na Alemanha, especialmente por Jakob Steiner, um especialista em métodos sintéticos, os quais podem ser encontrados através dos seus trabalhos. Em tais trabalhos praticamente não se percebe quase nenhum desenvolvimento algébrico, além disso, eles se tornaram poderosos instrumentos de descoberta e proporcionaram realizar uma visão melhor sobre a natureza e as propriedades de curvas (GOW, 1997, p.51).

Destaca-se, sobretudo, que os métodos sintéticos desenvolvidos por Poncelet e Chasles foram influenciadores de George Salmon, quando este escreveu A treatise on conic sections, em 1848, onde se encontram os primeiros resultados dessa nova geometria.

Mas em que consiste o método sintético? Uma possível resposta se encontra em Wilson (1903, p. 248) que afirma que o tratamento sintético depende essencialmente da concepção e da medida numérica da relação cruzada e que começa a partir do conhecimento que já se possui dos Elementos de Euclides. Wilson (1903) comenta ainda que os pilares da geometria projetiva sintética se edificaram no estilo claro de Poncelet, de Steiner e de Chasles, cabendo depois a Von Staudt de estabelecer a fundamentação axiomática dessa geometria.

\footnotetext{
${ }^{19}$ Princípio de continuidade também chamado de princípio da permanência das relações matemáticas.

${ }^{20} \mathrm{O}$ referido trabalho de Poncelet foi publicado em dois volumes: o primeiro em 1862 e o segundo em 1864.
} 
De um ponto de vista puramente geométrico, os métodos sintéticos são considerados mais naturais, isto é, mais intuitivo e também, ao mesmo tempo, são considerados mais compactos sendo abrangentes (LORENAT, 2015, p. 157).

Assim as vantagens da metodologia sintética consistem em que seus processos são mais diretos e que a construção de uma cônica, propriamente dita, era um belo problema e exercício com relação ao ponto de vista do ensino. Além disso, as ideias introduzidas pelos conceitos das propriedades harmônicas, como a de polar, por exemplo, são compreendidas mais facilmente (WILSON, 1903, p. 252).

Tanto essas vantagens quanto em relação ao ponto de vista de ensino foram aplicados na matemática britânica como também se pode confirmar no comentário que Richards (1986) faz a respeito:

\section{"Os defensores da geometria primária argumentam que a manipulação dos símbolos algébricos era vazia e não era útil no treinamento de raciocínio dos jovens" (RICHARDS, 1986, p. 306) ${ }^{21}$.}

No que diz respeito aos conceitos abordados através da perspectiva sintética, encontram-se, por exemplo, os seguintes teoremas e ideias elementares da geometria projetiva: o princípio da dualidade, o teorema de Desargues, a construção dos elementos harmônicos, a teoria das relações projetivas entre os pontos, os elementos da teoria de colineação e correlação entre os pontos e retas (WILSON, 1903, p. 250).

Em termos projetivos, há a definição das cônicas e dos teoremas usuais com relação aos quadriláteros inscritos e circunscritos, os teoremas de Pascal e de Brianchon e a teoria de pólos e polares, propriedades que completam os elementos básicos do estudo das seções cônicas sob o ponto de vista sintético.

Muitos desses conceitos, que usavam a perspectiva sintética, dados à geometria projetiva através de Poncelet e Steiner, baseavam-se em propriedades métricas. Von Staudt, em Geometrie der Lage, de 1847, libertou a geometria projetiva de qualquer relação métrica, mostrando que as coordenadas na reta projetivas podiam ser construídas a partir do conceito de divisão harmônica.

É fato que os métodos sintéticos também apresentavam suas desvantagens e quando esses inconvenientes surgiam, por sorte, poderiam ser resolvidos utilizando-se de alguma aplicação analítica. Wilson (1903, p. 249) relata ainda que a "morte" do desenvolvimento sintético ocorre efetivamente com a publicação dos últimos métodos, que se encontram no trabalho de Von Staudt de 1847 e que até mesmo os apreciadores desses métodos reconhecem que já estavam ultrapassados.

Esses últimos resultados de Von Staudt são os mais importantes e mais bonitos porque eles lidam diretamente com as ideias essenciais da geometria.

21 "The advocates of the primacy of geometry in education argued that manipulating empty algebraic symbols was not helpful in training young men to think."

RBHM, Vol. 19, n 38, p. 45-81, 2019 


\subsection{Da visão analítica}

De modo análogo à parte 4.2, o objetivo aqui será de procurar esclarecer o tratamento analítico na geometria, sobretudo, na projetiva. A influência analítica na geometria projetiva pode ser identificada na França através do Aperçu Historique, de 1837, de Michel Chasles e na Alemanha com os trabalhos de Möbius e de Plücker. Este se destaca, sobretudo, com o seu trabalho System der Analytischen Geometrie, de 1835.

Assim como anteriormente foi feito um destaque para o trabalho de Chasles, aqui também destaca o fato de que embora ele tenha contribuído significativamente para o desenvolvimento sintético, pode-se encontrar também em seus trabalhos contribuições com um tratamento analítico para a geometria.

Já a importância dos trabalhos de Plücker é devida, principalmente, a elaboração do conceito de coordenadas homogêneas ${ }^{22}$ e ao tratamento analítico das propriedades projetivas. Destacam duas vantagens desse novo método: a primeira é a idéia de que as curvas e as superfícies são mais gerais e, a segunda, de que os pontos imaginários, agora, aparecem nas equações algébricas. Uma das belas aplicações desse método de Plücker foi utilizada para estudar as curvas de terceira ordem. Quanto a Möbius, seu destaque é dado por causa do seu trabalho sobre o cálculo baricêntrico ${ }^{23}$ (MANSION, 1873, p. 314).

Dessa forma, considera-se que a introdução de uma visão analítica das propriedades projetivas, dadas pelas coordenadas homogêneas, ocorre a partir dos trabalhos de Möbius e de Plücker.

Com relação a essa nova visão analítica das propriedades projetivas dadas pelas coordenadas homogêneas, há um desenvolvimento interessante do sistema de coordenadas apresentado por Plücker em 1829, que, devida a simetria entre variáveis e parâmetros, a mesma equação pode reprentar uma reta ou um feixe de retas que passa por um ponto, assim como uma curva pode ser considerada ou como o lugar de seus pontos ou como a envoltória de suas tangentes, fornecendo, dessa forma, a base da demonstração analítica de Plücker do princípio da dualidade (LORENAT, 2015, pp. 167-168).

A sua obra Analytisch-geometrische Entwicklungen foi publicada em dois volumes, primeiro em 1828 e depois em 1831. No primeiro volume aparece o primeiro tratamento extenso do método da notação abreviada ${ }^{24}$, embora este já tivesse sido empregado antes por Gabriel Lamé e Étienne Bobillier, assim como alguns teoremas aparentemente complexos, do ponto de vista algébrico, como o teorema de Desargues e o teorema do hexagrama místico de Pascal, que podem ser demonstrados de maneira muito mais breve e clara com o auxílio da notação abreviada.

No segundo volume dessa obra, encontra-se uma apresentação das coordenadas homogêneas dos pontos de um plano. As coordenadas homogêneas de um ponto $\mathrm{P}$ de

\footnotetext{
${ }^{22}$ Coordenadas Homogêneas são as coordenadas utilizadas para indicar um elemento do plano projetivo e a sua notação clássica é dada da forma: $\bar{v}=\left(v_{1}: v_{2}: v_{3}\right)$, (ANDRADE; BARROS, 2010).

${ }^{23}$ A obra Barycentrische Calcul de A. F. Möbius publicada em 1827 já trazia um sistema de coordenadas homogêneas, as chamadas coordenadas baricêntricas.

${ }^{24}$ A ideia da notação abreviada reside na representação de expressões longas por letras únicas é o princípio fundamental: Se $\alpha=0$ e $\beta=0$ são equações duas curvas, então $\alpha+\beta=0=$, onde $\alpha$ e $\beta$ são funções quaisquer de $x$ e $y$, é uma curva que passa pelos pontos de interseção das curvas $\alpha=0$ e $\beta=0$.
} 
coordenadas cartesianas $(\mathrm{X}, \mathrm{Y})$ são definidas como qualquer terno ordenado $(\mathrm{x}, \mathrm{y}, \mathrm{t})$ tal que:

$$
\left\{\begin{array}{l}
\mathrm{X}=\frac{x}{t} \\
\mathrm{Y}=\frac{y}{t}
\end{array}\right.
$$

Segue-se que os ternos $(x, y, t)$ e $(k x, k y, k t)$ representam o mesmo ponto. A expressão "homogênea" provém do fato de que, quando se converte a equação $\mathrm{f}(\mathrm{X}, \mathrm{Y})=0$ de uma curva algébrica em coordenadas cartesianas à forma $\mathrm{f}\left(\frac{x}{t}, \frac{y}{t}\right)=0$, todos os termos da nova equação possuem o mesmo grau em relação às novas variáveis. A importância que o sistema de coordenadas homogêneas proporcionou foi a questão do terno do tipo $(x, y, 0)$, que não tem um correspondente em coordenadas cartesianas, poder representar um "ponto no infinito", de maneira que os pontos ideais no infinito ${ }^{25}$ de Kepler, Desargues e Poncelet passaram a ter uma representação num sistema de coordenadas.

As coordenadas homogêneas fornecem um instrumento perfeito para a exploração da geometria projetiva, a qual requer tanto os pontos finitos quanto os pontos infinitos do plano para abordar, por exemplo, os problemas de projeção (VOLKERT, 2019).

Foi um desenvolvimento extremamente importante, pois, ao apresentar as geometrias não euclidianas a partir da chamada geometria projetiva, pôde-se introduzir uma nova hierarquia entre as novas geometrias (MENGHINI, 2019).

Esses novos métodos foram rapidamente adotados pelos matemáticos britânicos, em particular, por Arthur Cayley que em 1859 mostrou como aplicar esses métodos para definir as métricas não euclidianas a partir das coordenadas homogêneas.

Um exemplo representativo dessa abordagem é o artigo de Cayley de 1843, Demonstration of Pascal's theorem, publicado no quarto volume do The Cambridge Mathematical Journal, na seção intitulada Plane Geometry, onde ele realiza uma demonstração analítica do teorema de Pascal, utilizando as equações homogêneas e trabalhando no espaço projetivo.

Levanta-se também a questão: em que consiste o tratamento analítico? Essencialmente, os métodos analíticos residem no desenvolvimento adequado de um sistema de coordenadas adotado a partir da escolha de um referencial apropriado para, enfim, conseguir estabelecer as equações e, desse modo, chegar às propriedades geométricas.

As vantagens que os métodos analíticos apresentam estão, exatamente, no poder de expressão da generalidade, uma vez que todos os problemas são resolvidos por procedimentos uniformes, ao passo que, nos métodos sintéticos, cada problema depende da figura particular considerada. Através do uso do sistema de coordenadas homogêneas, possibilita-se trabalhar as propriedades projetivas de forma algébrica, conforme a teoria de Plücker das curvas algébricas. Destaca-se também o interesse por problemas geométricos na perspectiva analítica de Chasles, na utilização das propriedades projetivas, e de transformações, como as homografias e, por último, o uso do determinante para realizar a

\footnotetext{
${ }^{25}$ Por exemplo, a equação $t=0$ é então a equação de uma reta ideal no infinito.
} 
interpretação geométrica de pontos alinhados e de pontos pertencentes ao mesmo plano (LORENAT, 2015, p. 158).

A geometria analítica, quando usada adequadamente, não perde para a geometria sintética em elegância e simplicidade. Quanto à desvantagem, os métodos analíticos perdem a facilidade do processo, quando o sistema de coordenadas não é escolhido adequadamente, podendo ocasionar excessivos cálculos algébricos. Outra desvantagem ocorre quando do não uso das notações abreviadas, as expressões podem tornar-se longas demais.

\subsection{A rivalidade}

A rivalidade que se estabeleceu entre a abordagem analítica e sintética pode ser conferida com o apontamento que Richards (1986, p. 301) faz, mostrando que desde o início, já no século XVIII, o desenvolvimento analítico se mostrava promissor, porém ainda não havia uma percepção clara dos motivos pelos quais os métodos analíticos levariam a resultados verdadeiros, pois pareciam ser meramente simbólicos e remotos.

Em contrapartida, Richards também aponta que o método sintético era considerado fundamentalmente seguro, visto que os processos eram claros e os objetos facilmente identificados, porém o seu ponto desfavorável era a falta da generalidade da análise.

Lorenat $(2015$, p. 157) também comenta a respeito desta rivalidade entre a geometria sintética e analítica delineada no início do século XIX era tema popular e que se estendeu até o do século XX.

Num primeiro momento, percebeu-se uma tentativa de se harmonizar a relação entre as duas perspectivas. Durante o começo do século XIX, como o interesse pelas metodologias analíticas foi tão significativo que um prêmio foi oferecido pela Sociedade Científica de Bordeaux, em 1813, para o melhor ensaio caracterizando a síntese e a análise e a influência exercida por cada uma delas.

Esses ensaios exprimiam a esperança de que houvesse uma reconciliação entre os dois campos, porém seis anos depois, a rivalidade recomeçou e tornou-se cada vez mais amarga. O curioso dessa rivalidade se destaca pelas figuras de Poncelet e Gergonne, pois ambos foram estudantes de Monge, o qual era igualmente adepto da geometria analítica e da sintética.

Com o falecimento de Monge em 1818, Poncelet e Gergonne publicaram artigos nos Annales de Gergonne, em que este defendia os métodos analíticos e aquele, a superioridade da geometria sintética.

Uma das evidências do confronto entre esses dois campos se percebe em 1826, no recém-descoberto princípio da dualidade. Enquanto Gergonne estava convencido de que os métodos analíticos mostravam que a permuta entre os termos, como ponto por reta, era universalmente válida. Em contrapartida, Poncelet afirmava que ele foi o primeiro a descobrir a dualidade e que o princípio era uma consequência das relações na geometria pura ente pólo e polar com relação a uma cônica.

Outra evidência desse confronto se percebe quando Steiner ameaçou os editores da prestigiosa revista de matemática pura e aplicada, o jornal de Crelle, de não publicar mais seus trabalhos caso continuasse aceitando os trabalhos de Plücker (LORENAT, 2016). 
Identifica-se dessa forma que na primeira metade do século XIX, estabeleceu-se uma grande controvérsia entre os geômetras sintéticos e os geômetras analíticos. As objeções que foram colocadas à geometria analítica eram do seguinte tipo: será que realmente é geometria? Os métodos são puramente algébricos e os resultados também, com os quais o significado geométrico não é considerado. A relação entre o ponto de partida e o ponto de chegada é perdida no processo de pequenos passos algébricos cujo significado geométrico não é claro. O método geométrico puro é mais simples e mais intuitivo em suas demonstrações. A geometria é a verdade sobre o mundo real; no entanto, análise e álgebra não são verdades em si.

Em resposta a essa crítica, percebe-se que os defensores dos métodos analíticos argumentavam que a vantagem da análise consistia na sua potencial generalização do método, uma vez que todos os problemas podem ser resolvidos por meio de processos convencionais, ao passo que nos métodos sintéticos, cada problema depende da figura em particular considerada.

Após essa fase de conflitos mais latentes, surgiram figuras notáveis como Arthur Cayley, James Joseph Sylvester e George Salmon, geômetras britânicos analíticos, que contribuíram substancialmente para os desenvolvimentos analíticos da teoria e que adotaram a visão analítica de Möbius e Plücker por volta da década 1840 a1850.

Lorenat (2016) ressalta que a partir daí a geometria projetiva entrou num estágio de maturidade cujo nome-chave foi Von Staudt, que mostrava como os métodos projetivos englobavam os métodos da geometria euclidiana. Mais tarde, já no final do século XIX, Felix Klein completou o trabalho de Staudt através da teoria dos grupos de transformação em seu famoso Erlangen Programm.

Mostrando a importância que esse trabalho de Klein proporcionou para a relação entre as duas metodologias, Wilson comentou:

"Desde a publicação do Programa de Erlangen, de Klein, em 1872, não há razão para que os geômetras não reconheçam distintamente - embora muitas vezes demorem a fazê-lo - que o fundamental, ao lado do axioma, é a transformação ou grupos de transformações. Na geometria do plano projetivo, seja o tratamento analítico ou sintético, o importante é a aplicação do grupo de colineações associada às correlações" (WILSON, 1903, p. 252) ${ }^{26}$

Dessa forma, embora de forma gradativa, percebe-se uma relativa trégua entre as duas perspectivas, e, além disso, a possibilidade de estabelecer-se um trabalho conjunto com a presença dos dois tratamentos.

O resultado que merece ser destacado se trata das aplicações geométricas ao tratamento analítico como se encontra nas conclusões da Sexta Memória de Cayley, que

26 "since the publication of Klein's Erlangen Programm in 1872, there has been no reason why geometers should not recognize distinctly - although they have ofen been slow to do so - that the fundamental thing, next to the axiom, is the transformation or groups of transformations."

RBHM, Vol. 19, n 38, p. 45-81, 2019 
ressalta que a geometria euclidiana seria um caso particular da geometria projetiva, e esta sendo então a mais geral.

Esse resultado foi significativamente inovador nos meados do século XIX, visto que a geometria projetiva, que surgia nesse período, considerava-se derivada da geometria euclidiana.

\section{As principais produções de George Salmon}

O objetivo aqui é fazer uma apresentação dos principais trabalhos no contexto da matemática feitos por George Salmon que o fizeram destacar-se no cenário matemático do século XIX.

Seu reconhecimento se deve, sobretudo, pelos quatro tratados que ele escreveu entre 1848 a 1862: o primeiro tratado foi A treatise on conic section (1848), com várias edições, seguido pelos tratados A treatise on the higher plane curves (1852) e Lessons introductory to the modern higher álgebra (1859), e, o último, A treatise on the analytic geometry of three dimensions (1862).

Nesses tratados foram publicados os recentes resultados tanto da álgebra quanto da geometria "moderna" daquele momento. O que se pode perceber nas obras de Salmon é a diferença notável entre a perspectiva sintética do primeiro tratado, e a perspectiva analítica dos demais. As principais produções de Salmon serão apresentadas em duas partes: a primeira faz uma abordagem sobre a produção dos seus quatro tratados, apontando os recem resultados que estavam sendo publicados na época, enquanto a segunda enumera os artigos de Salmon publicados em revista.

\subsection{Seus quatro tratados}

A importância de George Salmon se deve ao fato dele ter exercido uma grande influência tanto sobre a pesquisa quanto sobre o ensino de matemática na Europa e na América principalmente a partir da segunda metade do século XIX. Essa influência é, particularmente, consequência das publicações dos quatro tratados que ele escreveu no período compreendido entre 1848 a 1862 enquanto esteve atuante no departamento de matemática da Trinity College em Dublin (GOW, 1997, p. 26). Gow comenta ainda que além desse efeito de divulgação dos novos resultados, suas obras também se tornaram referências no sentido de disseminar e de popularizar a pesquisa contemporânea em álgebra e em geometria naquele momento.

Como já destacado nesse artigo, a matemática britânica passava por mudanças, as quais naturalmente faziam os matemáticos daquele momente a passarem por transformações, principalmente, nos aspectos de perspectivas sintéticas e analíticas. Em particular, não foi diferente com Salmon, pois se percebe na publicação de A treatise on conic sections em 1848 que nos últimos capítulos se encontram os recentes resultados da geometria daquela época, como, por exemplo, os métodos das notações abreviadas e os métodos geométricos, o princípio da dualidade, as propriedades harmônicas e anarmônicas 
das cônicas e os métodos das projeções, abordados sob uma perspectiva sintética, principalmente, sob a influência de Poncelet e de Chasles (ibid., p. 45).

A treatise on the higher plane Curves tinha por objetivo declarado ser um tratado de geometria analítica. Tinha como proposta abordar os conceitos analíticos do plano, como, por exemplo, coordenadas, propriedades gerais das curvas algébricas, curvas do terceiro e do quarto graus, curvas transcendentais e que se destinava a um público mais avançado do que o seu primeiro tratado (ibid., p. 54).

Nesse tratado ainda se percebe a influência de alguns resultados de Poncelet e Chasles, mas a novidade se manifesta com a inclusão dos resultados de Plücker, como o próprio Salmon destaca em seu prefácio:

"Os trabalhos de Plücker sobre geometria analítica eu não os conhecia quando a primeira edição do tratado sobre as seções cônicas foi publicada, mas tenho repetidamente tido a oportunidade de reconhecer minhas obrigações para com eles nas páginas seguintes" (SALMON, 1952, p. V). ${ }^{27}$

O tratado ainda faz uma indicação com relação ao interesse de Salmon pela teoria dos invariantes, a qual será o conteúdo do seu próximo trabalho, Lessons introductory to the modern higher algebra, de 1859.

O conteúdo desse terceiro tratado expõe a teoria dos invariantes, ou como Salmon a chamou: a álgebra das transformações lineares. O objetivo de Salmon ao escrever esse trabalho foi de introduzir aos leitores os estudos sobre a teoria dos invariantes, a qual foi desenvolvida entre a década de 1840 a 1850 por Boole, Sylvester e Cayley (GOW, 1997, p. 57). A essência dessa teoria se dá com a combinação dos resultados da álgebra linear associados aos da geometria algébrica.

Nesse trabalho há dezessete lições que abordam, por exemplo, tópicos como determinantes, teoria da eliminação, discriminantes, quantics, invariantes e covariantes, formas canônicas e transformações ortogonais.

$\mathrm{O}$ quarto e último tratado de Salmon foi A treatise on the analytic geometry of three dimensions publicado em 1862. Esse trabalho foi o maior em relação aos três anteriores, de modo que a sua primeira edição foi publicada com 465 páginas e as edições seguintes ainda foram bem maiores. (ibid., p. 62). O tratado se divide em quinze capítulos que abordam conteúdos como: o ponto, a interpretação das equações, o plano, as propriedades gerais das quádricas, os métodos de notação abreviada, a teoria geral das superfícies. Nesse trabalho pode-se encontrar a teoria das vinte e sete retas sobre uma superfície cúbica, a qual é considerada o resultado mais expressivo de Salmon (ibid., pp. 62-63).

Esses quatro tratados, A treatise on conic sections, A treatise on the higher plane curves, Lessons introductory to the modern higher algebra, A treatise on the analytic geometry of three dimensions não só deram um tratamento abrangente dentro das suas

27 "Plücker's works on Analytic Geometry I was not acquainted with when the first edition of the conics was published, but I have repeatedly had occasion to acknowledge my obligations to them in the following pages. "

RBHM, Vol. 19, n 38, p. 45-81, 2019 
respectivas áreas, como também serviram de modelo pela clareza da escrita e a elegância de estilo, as quais se tornaram as características padrão de um livro-texto na Grâ-Bretanha (MCCONNELL, 1981, p. 86).

Ainda em relação aos tratados de Salmon, os mesmos foram traduzidos para todos os idiomas da Europa Ocidental e passaram por muitas edições, cada uma incorporando os novos desenvolvimentos. Além disso, seus tratados também permaneceram por muitos anos servindo de livros didáticos em suas respectivas áreas.

Dentre as traduções realizadas nas obras de Salmon, destacamos as traduções feitas para o alemão realizadas pelo matemático Wilhelm Fiedler cujas contribuições foram altamente consideradas para o ensino de geometria na Alemanha na segunda metade do século XIX.

\subsection{Seus artigos em matemática}

Serão abordadas a seguir as contribuições de Salmon quanto às publicações dos seus artigos em matemática nos mais diversos periódicos renomados tanto britânicos quanto do continente europeu.

Salmon além de ensinar matemática e de escrever seus quatro tratados, contribuiu com seus artigos matemáticos, principalmente sobre superfícies. A publicação deles ocorreu em vários periódicos, como o The Cambridge and Dublin Mathematical Journal, The Philosophical Magazine e The Proceedings of the Royal Irish Academy. E nos jornais de mais prestígio como os periódicos Jornal de Crelle, Transactions of the Royal Irish Academy, e The Philosophical Transactions of the Royal Society (MOLLAN, 2007, p. 774).

A relação efetiva dos artigos de pesquisa em matemática de Salmon se encontra no Royal Society's Catalogue of Scientific Paper (GOW, 1997, p. 34). Nota-se pelos títulos dos artigos que quase todos os seus trabalhos foram dedicados a assuntos da geometria. Seus artigos são, na maioria, não muito extensos, geralmente não sendo mais do que notas de pesquisas ou anúncios. Seu último trabalho em artigo de pesquisa foi publicado em 1873, iniciando ainda a teoria elementar dos números e a sua dedicação sobre o assunto foi bastante pequena, uma vez que esses estudos eram bem originais e bastante difíceis no momento em que Salmon os escreveu, sendo esses últimos resultados essencialmente triviais (ibid., p.38).

A respeito da pesquisa de Salmon, Joly comenta:

"Seria muito injusto com Salmon, julgar suas contribuições para a matemática apenas pelos seus artigos. Ele tinha uma grande aversão ao problema físico de escrever; Ele modestamente comunicava suas descobertas a amigos, ou reservava-as para incorporar em seus livros, de modo que é 
uma questão de extrema dificuldade dizer o que de fato é referente a Salmon" (JOLY, 1905, p. 354). ${ }^{28}$

Com relação a alguns dos resultados encontrados nos artigos de Salmon, o seu artigo de 1856, On the degree of a surface reciprocal to a given one, mostra, por exemplo, que Salmon se utiliza de polinômios homogêneos e trata de superfícies e propriedades projetivas (SALMON, 1856, p. 468).

Salmon publica também dois artigos no The Cambridge and Dublin Mathematical Journal, de 1848, On the condition that a plane should touch a surface along a curve line $\mathrm{e}$ On the number of normals which can be drawn from a given point to a given surface. Esses dois artigos fazem referências quanto ao uso das equações diferenciais, sob uma perspectiva geométrica, relacionando-se através dos desenvolvimentos algébricos (SALMON, 1848, pp. 44-47).

Em seu artigo Théorèmes sur les courbes de troisième degré publicado no jornal de Crelle em 1852, Salmon apresenta três teoremas, sendo que, no último, utiliza uma definição de Plücker. Nesse artigo, Salmon se utiliza, mais uma vez, dos polinômios homogêneos e estuda algebricamente os diferentes casos de interseções de cônicas, entre outras propriedades ligadas aos métodos projetivos (SALMON, 1852, pp. 321-322).

Assim a relação entre os conteúdos publicados nos tratados de Salmon e também em grande parte de seus artigos mostra a importância que eles tiveram nas aplicações geométricas associadas à álgebra, trazendo os principais resultados da teoria projetiva das curvas envolvendo a geometria "moderna" daquele momento.

\section{O primeiro tratado de Salmon: A treatise on conics sections}

Um dos grandes fatores que motivou George Salmon a escrever os seus dois primeiros tratados se deve, sobretudo, quando ele lecionava na graduação da Trinity College de Dublin durante a década de 1840 (GOW, 1997, p. 30). Salmon, inicialmente, foi influenciado pelo o ambiente em que se encontrava visto que os desenvolvimentos dos resultados matemáticos sob a perspectiva sintética eram predominantes naquele momento.

Entretanto, com os avanços dos resultados analíticos que estavam ocorrendo naquela época juntamente com as influências de matemáticos, como Cayley e Sylvester, que já trabalhavam diversos resultados sob a perspectiva analítica, promoveram em Salmon uma mudança nos tratamentos dos seus trabalhos na matemática, e Salmon começou a publicar resultados sob a perspectiva analítica.

É exatamente nos dois primeiros tratados, A treatise on conic sections e A treatise on the higher plane curves, onde se percebe essa mudança em Salmon quanto ao tratamento adotado nas suas obras, sendo que o primeiro apresenta claramente a influência sintética oriunda dos trabalhos de Poncelet, Chasles e Steiner, enquanto o segundo apresenta a influência analítica de Cayley, Sylvester, Plücker e Möbius.

28 "It would be most unfair to Salmon to judge his contributions to mathematics by his papers alone. He had a great dislike to the physical trouble of writing; he modestly communicated his discoveries to friends, or reserved them for incorporation in his books, so that it is a matter of extreme difficulty to say how much is his."

RBHM, Vol. 19, nº 38, p. 45-81, 2019 
A partir dessa compreensão, será feita uma análise nos dois primeiros tratados de Salmon, destacando, sobretudo, as evidências que apontam a perspectiva adotada nos resultados, sintética no primeiro, analítica no segundo e, por fim, fazer uma comparação entre as duas obras.

\subsection{Uma análise de $A$ treatise on conic sections}

A análise do primeiro tratado de Salmon, o qual foi considerado sua obra mais famosa, na Grã-Bretanha, busca encontrar as motivações que o levaram a escrevê-lo, bem como mostrar os novos resultados que foram publicados na primeira edição.

Antes da publicação em 1848 do A treatise on conic sections de Salmon, os trabalhos que havia sobre cônicas eram os publicados por Henry P. Hamilton, cujo título era An analytical system of conic sections, em 1828, substituído mais tarde pela publicação de John Hymers, cujo título também era Conic Sections, em 1837 e publicado em 1839 (BALL, 1889, p. 130).

A história da publicação do tratado de Salmon é um pouco complicada. Isso se deve ao fato de que, na década de 1840, Salmon havia planejado escrever um tratado em dois volumes sobre geometria analítica, o qual pretendia utilizar na graduação da Trinity College de Dublin. O primeiro volume desse trabalho foi dividido em duas partes: a primeira parte consistia na abordagem da geometria analítica elementar e a segunda parte, consistia na abordagem da geometria das seções cônicas, com ênfase especial no desenvolvimento dos resultados da geometria "moderna" daquele momento. Já o segundo volume formou a terceira parte do tratado inicial, o qual fazia abordagem das curvas planas superiores. O resultado final desse planejamento foi, essencialmente, a publicação de $A$ treatise on conic sections em 1848 e, posteriormente, a publicação de A treatise on the higher plane curves em 1852 (GOW, 1997, p. 42).

Tanto na primeira edição de 1848 quanto na segunda de 1850 de A treatise on conic sections não havia prefácio, aparecendo somente a partir da terceira edição em 1855. Nesse prefácio, Salmon deixa claro que seu trabalho visava atrair a atenção dos estudantes para o estudo das cônicas que considerava extremamente útil para a matemática superior, sobretudo, por apresentar os novos métodos algébricos e geométricos desenvolvidos naquele período.

Com relação ainda ao primeiro prefácio de A treatise on conic sections da edição de 1855, destacam-se alguns trechos que chama atenção para alguns pontos em relação à preocupação que Salmon apresentou ao publicar o seu trabalho: o primeiro é com relação ao ensino.

"Tendo-me utilizado nos últimos cinco anos no ensino de Geometria Analítica para calouros, ganhei alguma experiência quanto aos pontos em que os alunos provavelmente sentem dificuldades" (SALMON, 1855, p. V) ${ }^{29}$

\footnotetext{
29 "Having myself used it for the last five years in teaching Analytic Geometry to beginners, I have gained some experience as to the points where learners are likely to feel difficulties"
} 
O segundo é com relação à publicação sistemática dos novos resultados naquele momento.

"Os capítulos finais tratam dos métodos algébricos $e$ geométricos que foram introduzidos nos últimos anos, mas dos quais nenhuma contribuição sistemática foi dada em qualquer trabalho elementar no momento em que a primeira edição deste Tratado foi publicada" (SALMON, 1855, p. V) ${ }^{30}$.

Pelo o que se pode perceber, de fato, em Salmon quando publicou a terceira edição de A treatise on conic sections, que ele se mostrava não só atento aos novos resultados, mas também se preocupava com a questão do ensino, principalmente, nas questões onde os alunos apresentavam suas dificuldades, buscando saná-las com a ilustração de diversos exemplos e também orientando o estudo partindo dos conceitos elementares da geometria para os iniciantes e para os mais avançados, indicando os capítulos com os novos resultados.

Salmon comenta o fato de não encontrar outras referências de publicações dos novos resultados durante entre 1848 e 1855 , período que compreendeu a primeira e terceira edição de A treatise on conic sections, o que promoveu, dessa forma, o papel fundamental de Salmon ao ser responsável de dar conta dos novos resultados, publicando-os em cada nova edição. Na terceira edição de 1855, não há apenas quatorze capítulos, mas quinze. Os capítulos de I a XIII tratam dos conceitos e das propriedades elementares da geometria plana. O capítulo XIV trata das notações abreviadas que na época era uma tentativa de dar conta das relações entre objetos geométricos através de uma simbologia, no espírito de Boole, Sylvester e Cayley. Convém dizer que essa simbologia se tornou rapidamente obsoleta e abandonada pelos críticos dos trabalhos de Salmon. E o Capítulo XV representa a grande inovação da terceira edição, ao dar conta da divulgação dos resultados recentes no domínio dos estudos da teoria das cônicas

\subsection{Os novos resultados}

A proposta do capítulo sobre as notações abreviadas ${ }^{31}$, cuja finalidade reside em representar longas expressões se utilizando de letras únicas, é apresentar o uso dessas notações em resultados como a prova das propriedades da razão anarmônicas das cônicas, o teorema de Brianchon, o teorema de Pascal e de Steiner, e o método de MacLaurin. O último capítulo sobre os métodos geométricos é o de maior importância de A treatise on conic sections, pois Salmon faz uma apresentação sistemática dos resultados da geometria da primeira

30 "The remaining chapters treat of the algebric and geometric methods which have been introduced into use of late years, but of which no systematic account had been given in any elementar work at the time that the first edition of this treatise was published"

${ }^{31}$ Para entender o uso da notação abreviada, considere, por exemplo, Se $\alpha(x, y)=0$ e $\beta(x, y)=0$ são duas curvas, então $u \alpha+v \beta=0$, onde $u$ e $v$ são constantes ou funções quaisquer de $x$ e $y$, e uma curva pelos pontos de intersecção das curvas $\alpha=0$ e $\beta=0$.

RBHM, Vol. 19, n 38, p. 45-81, 2019 
metade do século XIX, iniciando a conceituação dos métodos das polares recíprocas juntamente com o princípio da dualidade, cujo crédito é dado a Poncelet evidenciando a influência deste sobre Salmon. Sobre o método das polares recíprocas, Salmon escreveu em uma nota de rodapé:

"Este bonito método foi introduzido por Sr. Poncelet, cuja descrição será encontrada no início do quarto volume do jornal de Crelle" (SALMON, 1850, p. 251). ${ }^{32}$

No último capítulo ainda encontra um estudo das propriedades harmônicas e anarmônicas das cônicas, dessa vez influenciada pelos trabalhos de Michel Chasles, seguido dos conceitos de involuções e dos métodos dos infinitesimais. A parte final trata dos métodos das projeções, a qual novamente Salmon descreve em uma nota de rodapé fazendo referência a Poncelet:

“O método é a invenção de Sr. Poncelet. Veja seu Tratado das propriedades projetivas [Traité], publicado no ano de 1822. Fico feliz se o pequeno esboço aqui dado induz qualquer leitor a estudar uma obra, da qual talvez tenha derivado mais informações do que qualquer outra sobre a teoria das curvas", (SALMON, 1850, p. 297). ${ }^{33}$

Apesar da maior parte de A treatise on conic sections apresentar vários resultados sob um tratamento sintético derivados das obras de Poncelet e de Chasles, observa-se na edição de 1855 que Salmon já valorizava as vantantagens de usar as coordenadas homogêneas, como se pode observar no Artigo 61.

"A vantagem das coordenadas trilineares é que, enquanto nas coordenadas cartesianas (ou $x$ e y), a máxima simplificação que podemos introduzir é a escolha de duas das retas mais notáveis na figura para eixos de coordenadas, enquanto que podemos obter em coordenadas trilineares expressões ainda mais simples ao escolher três das retas mais notáveis para as retas de referência $\alpha$, $\beta$ e $\gamma$ " (SALMON, 1855, p. 59). ${ }^{34}$

\footnotetext{
32 "The beautiful method was introduced by M. Poncelet, whose account of it will be found at the commencement of the fourth volume of Crelle's Journal."

33 "This method is the invention of M. Poncelet. See his Traité des Propriétes Projectives, published in year 1822. I shall be glad if the slight sketch here given induces any reader to study a work, from which I have perhaps derived more information than from any other on the theory of curves."

34 "The advantage of trilinear coordinates is, that whereas in Cartesian (or $x$ and $y$ ) co-ordinates the utmost simplification we can introduce is by choosing two of the most remarkable lines in the figure for axes of coordinates, we can in trilinear coordinates obtain still more simple expressions by choosing three of the most remarkable lines for lines of reference $\alpha, \beta, \gamma$."
} 
Aliás, no Artigo 63, ${ }^{35}$ Salmon propõe expressar em coordenadas trilineares a equação da paralela à reta dada cuja equação é $\mathrm{A} \alpha+\mathrm{B} \beta+\mathrm{C} \gamma=0$. Em seguida, relaciona alguns exercícios que ressaltam as relações entre equações homogêneas e equações cartesianas, conteúdos característicos de A treatise on higher plane curves.

\subsection{Razão anarmônica e as involuções}

Salmon, em uma nota de rodapé, credita a descoberta das propriedades anarmônica das cônicas a Michel Chasles, encontradas em History of Geometry (SALMON, 1855, p. 271).

Salmon destaca ainda que as propriedades harmônicas e anarmônicas das cônicas admitem inúmeras aplicações na teoria de curvas, isto é, pode-se encontrar o uso das razões anarmônicas, associadas à teoria de pólos e de polares, assim como a teoria de involução e aos casos que frequentemente são considerados como os mais importantes, que ocorrem quando um dos quatro pontos da reta se encontra no infinito.

Assim, o Artigo 324, Salmon destaca o caso da relação anarmônica dos quatros pontos, sendo um localizado no infinito. O artigo diz que a razão anarmônica de quatros pontos $\mathrm{A}, \mathrm{B}, \mathrm{C}, \mathrm{D}$ é, em geral, igual a $\frac{A B \cdot C D}{A D \cdot B C}$ e, se o ponto $\mathrm{D}$ estiver a uma distância no infinito, a razão $\frac{C D}{A D}=1$, de modo que a razão anarmônica se torne simplesmente $\frac{A B}{B C}$ (SALMON, 1855, p. 271).

Salmon descreve, a partir do Artigo 329, uma estrutura conceitual do que venha a ser um sistema de involução, usando uma abordagem totalmente sintética ao mostrar a relação harmônica entre os pontos dos dois sistemas dados (SALMON, 1855, pp. 275-276).

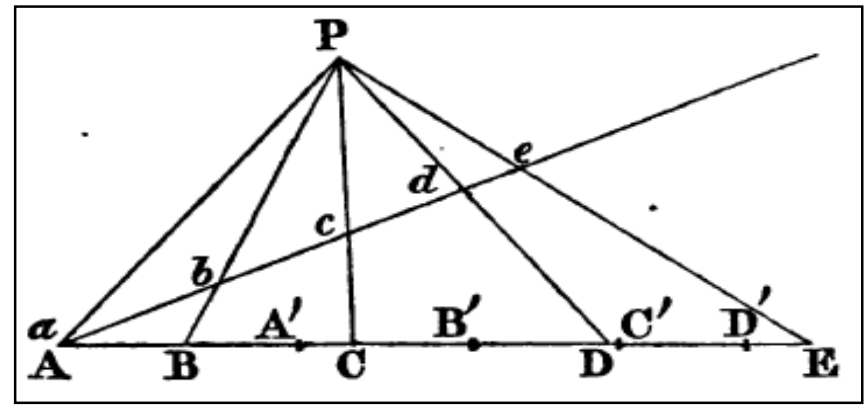

Figura 2: Sistemas de pontos em involução.

O sistema de três pares de pontos AA', BB', CC' é dito estar em involução se a razão harmônica de quatro pontos é igual à razão dos pontos conjugados. Assim, por exemplos, se os quatro pontos considerados são $A B B^{\prime} A$ ' e os seus respectivos são conjugados $A$ ' $B$ ' $B A$, a razão harmônica dos quatro primeiros deve se igual à razão harmônica dos quatro últimos.

35 "To express in trilinear coordinates the equation of the parallel to a given line $A \alpha+B \beta+C \gamma=0$.

RBHM, Vol. 19, no 38, p. 45-81, 2019 


\subsection{Polares recíprocas}

Os conceitos das polares recíprocas, os quais integravam a relação dos novos resultados publicados em A treatise on conic sections, foram introduzidos por Salmon, abordados sob uma perspectiva sintética, cuja prentensão era de mostrar que o uso desses métodos geométricos consistia em ser parte essencial em qualquer trabalho dedicado à teoria das curvas (SALMON, 1855, p. 253).

No Artigo 305, Salmon mostra a relação da dualidade entre o teorema de Briachon e o teorema de Pascal, o principal exemplo desse princípio, comentando que os respectivos resultados são recíprocos um do outro (ibid., p. 255).

O referido artigo diz: sejam $S$ e $s$ duas seções cônicas, o modo de obter um teorema do outro é usando esse método. "Se um hexágono inscrito em $S$, cujos vértices são A, B, C, D, E, F, então os pontos de interseção das retas que passam AD, BE, CF estão na mesma reta" (teorema de Pascal) e "Se um hexágono circunscrito em $s$, cujos vértices são $a$, $b, c, d, e, f$, então as retas $a d, b e, c f$ se encontrarão no mesmo ponto" (teorema de Brianchon), então ao fazer a troca dos termos pontos por retas, de fato, obtém-se o segundo teorema a partir do primeiro.

\subsection{Método dos infinitesimais}

Ao iniciar a parte do metódo dos infinitesimais, Salmon procura mostrar a importância do cálculo diferencial que permite traçar tangentes a curvas e determinar o valor de áreas e arcos. Destaca ainda como esses problemas eram abordados antes do desenvolvimento desse método e chama atenção que os métodos geométricos não são importantes apenas por uma questão de ponto de vista histórico, mas também porque eles fornecem soluções para algumas questões mais concisa e simples do que aquelas fornecidas pela ánalise (ibid., p. 289).

Mais uma evidência para reforçar o seu tratamento sintético se encontra no desenvolvimento do terceiro exemplo do Artigo $348^{36}$ para se determinar a área do círculo usando os métodos infinitesimais, onde Salmon utiliza um método de exaustão comparável ao de Arquimedes, para determinar que a área seja igual ao produto do raio pelo semiperímetro do círculo (ibid., pp. 290-291).

\subsection{Método das projeções}

Ao introduzir este novo conceito, Salmon comenta que o método das projeções exigirá um conhecimento da geometria de três dimensões, ainda que o método seja aplicado para geometria plana (SALMON, 1855, p. 299).

Salmon comenta também que a particularidade de tal método percorre um caminho exatamente oposto, onde a partir de um determinado teorema, pode-se inferir um resultado geral, diferentemente de obter teoremas particulares a partir de um enunciado geral.

${ }^{36}$ Ex. 3. "The area of a circle is equal to the radius multiplied by the semicircunference." 
No artigo 360, Salmon apresenta uma definição para o plano de projeção, mostrando que qualquer ponto de uma dada figura, corresponderá a um ponto de outra figura projetado no plano, e ainda que uma reta sempre seja projetada em outra reta.

No artigo 361, comenta que qualquer curva sempre será projetada em outra curva de mesmo grau. No artigo 363, que as propriedades não envolvem a métrica, não havendo relações de medidas de ângulos ou de segmentos. E no artigo 364 Salmon introduz o termo "propriedades projetivas" propriamente dito, cujo resultado mostra que se uma propriedade for verdadeira para uma figura, também será verdadeira para sua projeção (ibid., pp. 299$301)$.

Além disso, observa-se o tratamento sintético dado aos resultados dessa parte, como se pode encontrar no artigo $365^{37}$ : para demonstrar qualquer propriedade projetiva de qualquer figura, é suficiente demonstrá-la para uma figura mais simples na qual a figura dada pode ser projetada; por exemplo, para um caso em que uma reta da figura dada está no infinito. Assim, se for necessário investigar as propriedades harmônicas de um quadrilátero ABCD completo, cujos lados opostos se cruzam em E, F, e a interseção das diagonais é G, podem-se reduzir todos os pontos dessa figura em qualquer ponto no espaço $\mathrm{O}$, e cortar as retas de junção em qualquer plano paralelo a OEF, então EF será projetado no infinito, obtendo um novo quadrilátero, cujos lados $A^{\prime} B^{\prime}, C^{\prime} D^{\prime}$ se cruzam em no ponto $E^{\prime}$ no infinito e são paralelos; enquanto $A^{\prime} D^{\prime}, B^{\prime} C^{\prime}$ se cruzam em um ponto $F^{\prime}$ no infinito e também são paralelos (ibid., p. 302).

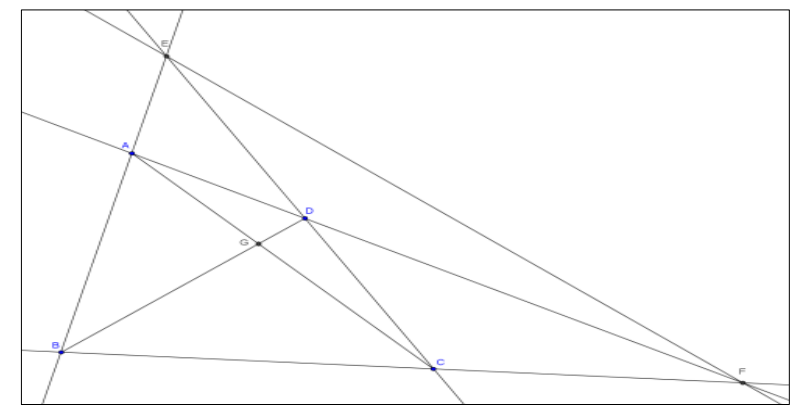

Figura 3: o caso das propriedades harmônicas de um quadrilátero ABCD completo

37 "If we wish to demonstrate any projective property of any figure, it is sufficient to demonstrate it for the simplest figure into which the given figure can be projected; $e$. g. for one in which any line of the given figure is at an infinite distance. Thus, if were required to investigate the harmonic properties of a complete quadrilateral $A B C D$, whose opposite sides intersect in $E, F$, and the intersection of whose diagonals is $G$, we may hoin all the points of this figure to any point in space $O$, and cut the joining lines by any plane parallel to $O E F$, then $E F$ is projected to infinity, and we have a new quadrilateral, whose sides ab, cd intersect at e at infinity, that is, are parallel; while $a d, b c$ intersect in a point $f$ at infinity, or are also parallel."

RBHM, Vol. 19, n 38, p. 45-81, 2019 


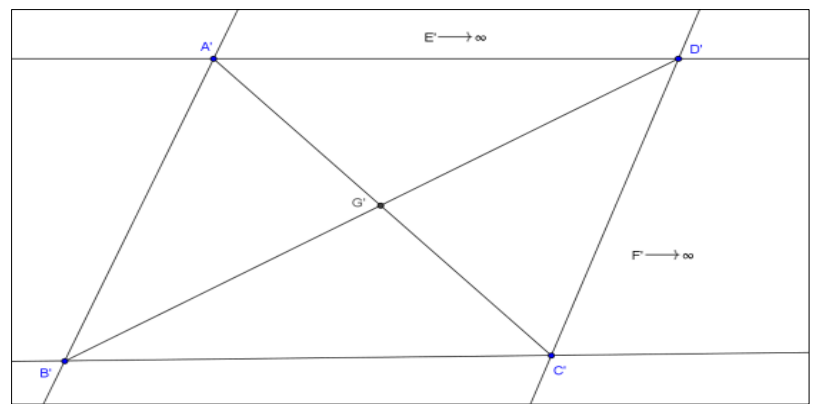

Figura 4: novo quadrilátero obtido, cujos lados $A$ ' $B$ ', $C^{\prime} D^{\prime}$ se cruzam em no ponto $E$ ' no infinito

Todos esses resultados podem ser vistos, por exemplo, na introdução da Quinta Memória sobre quantics, onde Cayley pretendia compor sistemas de duas ou mais quádricas e os resultados das teorias da relação harmônica e da involução. Considera também quádricas binárias bipartidas e sua relação com a chamada teoria da homografia, ou relação anarmônica (CAYLEY, 1858b, p. 527). Mas o trabalho de Cayley ao contrário de Salmon é puramente analítico.

\section{O segundo tratado de Salmon: A treatise on the higher plane curves}

A partir do segundo tratado de Salmon, A treatise on the higher plane curves, de 1852, já se percebe nitidamente uma mudança na escolha do tratamento adotado, passando a ser mais analítico. Com relação a essa mudança, o próprio Salmon destaca no prefácio dessa obra, que além de ter recebido diversas contribuições de Cayley, também se nota a influência dos resultados de Plücker, os quais eram desconhecidos até a edição do seu primeiro tratado (SALMON, 1852, p. v). Ainda no prefácio, Salmon destaca que além das influências dos, até então, desconhecidos trabalhos de Plücker, A treatise on the higher plane Curves recebeu influências ainda dos trabalhos de Poncelet e de Chasles, porém sua nova abordagem analítica era clara, sobretudo, devida aos novos resultados publicados nos artigos dos jornais de Crelle e Liouville e no Cambrigde and Dublin Mathematics.

Em relação às contribuições de Cayley, Salmon também registra no prefácio que tais contribuições se deram essencialmente no primeiro capítulo, sobre coordenadas, bem como com vários artigos para as edições seguintes. Salmon também incorporou no seu trabalho vários manuscritos não publicados por Cayley, como a teoria do envelope (ibid., $\mathrm{p}$. ix).

Para entender essa nova abordagem, a adoção do tratamento analítico, deduz-se que essa influência pode ser constatada através dos primeiros artigos de Cayley, os quais demonstram o quanto conhecia a respeito dos trabalhos dos matemáticos continentais como Lagrange, Jacobi, M. Chasles, Plücker, entre outros. Tal fato ajuda a entender a expressiva ajuda de Cayley nesse tratado e também permite Salmon optar para esses resultados de caráter analítico, como no uso das coordenadas homogêneas. 
A treatise on higher plane curves apresenta em seus sete capítulos conteúdos como coordenadas, propriedades gerais das curvas algébricas, curvas do terceiro grau, curvas do quarto grau, curvas transcendentais, métodos gerais e aplicações do cálculo de integral, uma vez que Salmon sinaliza no prefácio que sua intenção era de escrever um livro de referência para uma matemática mais avançado relacionado a curvas de dimensões superiores.

Uma mudança ainda mais nítida sobre o estilo de abordagem aparece nas edições posteriores como podemos identificar no prefácio da terceira edição de 1879 (SALMON, 1879 , p. v).

Salmon relata que um dos motivos para esse tempo entre as edições é devido à impossibilidade do cargo que ele estava ocupando na ocasião e que quando a primeira edição do tratado foi editada, o desenvolvimento da álgebra superior moderna ainda era muito recente e que agora, anos depois de tomar conhecimento dos recentes resultadostinha percebido a necessidade de fazer uma nova edição com as novas descobertas que representavam o progresso da ciência, mantendo, porém, a memória do que já havia [tinha] sido escrito anteriormente.

Outro comentário de Salmon que chama a atenção é a respeito da fama de seus livros, que mesmo se passado alguns anos, esse tratado era o único trabalho em inglês que fazia uma abordagem sistemática da teoria moderna das curvas.

Salmon não realizou muitos esforços para escrever seu segundo tratado, de modo que não houve uma adesão a esse tratado o qual ainda permaneceu apenas na língua inglesa por muitos anos, não causando o mesmo impacto no ensino e na pesquisa como se percebeu nas outras obras (GOW, 1997, p. 54).

\subsection{Análise de $A$ Treatise on the higher plane curves e as influências de Plücker}

Salmon sinaliza que o conteúdo do primeiro capítulo foi desenvolvido principalmente sob a influência do sistema analítico ${ }^{38}$ de Plücker (SALMON, 1852, p. 16). Nos três primeiros artigos do capítulo, Salmon, além de fazer uma apresentação sobre os sistemas de coordenadas, faz um comparativo entre o sistema de cordenadas cartesianas e o sistema de coordenadas trilineares.

Tal comparação consiste em mostrar que tanto em um sistema quanto no outro, o grau da equação indica exatamente o grau da curva, enquanto a determinação da classe da curva não pode ser verificada diretamente.

Além disso, a diferença que esses sistemas apresentam está relacionada quanto à representação dos conceitos: enquanto no sistema de coordenadas cartesianas, a posição de um ponto é determinada por coordenadas e a reta representada por uma equação, no sistema de coordenadas trilineares, a posição de uma reta é representada por coordenadas, enquanto a posição de um ponto é indicada por uma equação (ibid., pp. 3-4). A dualidade entre retas e planos é perfeitamente simétrica.

No artigo $2^{\circ}$, Salmon apresenta a equação da reta escrita no sistema de coordenadas cartesianas: $A x+B y+C=0$, onde os coeficientes $A, B, C$ são conhecidos.

${ }^{38}$ Esse sistema se refere a sua publicação de 1828 cujo título original é System der Analytischen Geometrie.

RBHM, Vol. 19, n 38, p. 45-81, 2019 
Já no Artigo 3, a representação da reta, em termos de coordenadas trilineares, é dado pela suas distâncias $\alpha, \beta, \gamma$ a três pontos fixos, $\mathrm{A}, \mathrm{B}, \mathrm{C}$ dados.

Ainda no artigo $3^{\circ}$, Salmon também faz a representação de um ponto no sistema de coordenadas trilineares, em termo das distâncias $\alpha, \beta, \gamma$ :

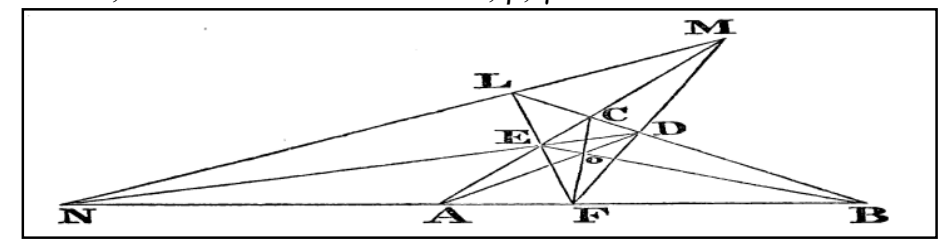

Figura 5 Ponto $O$ é construído a partir da razão anarmônica BD:DC, CE:AE e AF:FB

$$
\frac{\operatorname{sen} B O C}{O A} \alpha+\frac{\operatorname{sen} C O A}{O B} \beta+\frac{\operatorname{sen} A O B}{O C} \gamma=0
$$

Outro importante resultado foi a representação analítica de um ponto no infinito dada pelo sistema de coordenadas trilineares. Por exemplo, a equação geral de um ponto no infinito é dada pela equação $l \alpha+m \beta+v \gamma=0$. Salmon explica também como a partir da equação de duas cônicas, epressar a equação de um elemento do feixe gerado pelas duas cônicas.

\subsection{As coordenadas trilineares}

Outra influência de Plücker que ainda se observa no capítulo, refere-se à abordagem analítica feita por Salmon para classificar as cônicas.

$\mathrm{Na}$ introdução do artigo $7^{\circ}$, de acordo com o ponto de vista das coordenadas trilineares, com a inclusão dos pontos no infinito, a geometria projetiva permitiu uma uniformização das cônicas, não se distinguindo os diferentes tipos de cônicas - as elipses, as hipérboles e as parábolas - são indiscerníveis (SALMON, 1852, p. 8). Essa propriedade é devida à visão analítica do método das projeções.

Mas essa característica que permite a indistinção das cônicas difere da geometria sintética plana onde existe sempre uma transformação projetiva que leva uma cônica numa outra qualquer do mesmo tipo.

No presente sistema apresentado neste artigo $7^{\circ}$, Salmon estuda como através das coordenadas trilineares e o número de ponto no infinito, a cônica é uma parábola, ou uma elipse, ou ainda uma hipérbole.

Salmon comenta que esse tipo de sistema apresentado em termos das coordenadas trilineares é bem similar aos métodos dos cálculos baricêntricos apresentado por Möbius na sua publicação de $1827^{39}$.

${ }^{39} \mathrm{O}$ trabalho o qual Salmon se refere é o Barycentric Calculus publicado por Möbius em 1827. 


\subsection{Comparação entre $A$ treatise on conic sections e $A$ treatise on higher plane curves}

A partir do que foi exposto nas partes 6, 7.1 e 7.2 do nosso artigo, consegue-se realizar comparações entre os resultados publicados nos dois primeiros tratados de George Salmon, permitindo perceber que de uma obra para a outra, de fato, foi possível identificar uma mudança na abordagem adotada, no primeiro momento estava fortemente influenciada pelos métodos sintéticos de Poncelet e de Chasles e, no segundo, pelos métodos analíticos de Plücker e de Cayley.

Um exemplo dessa comparação pode ser encontrado no próprio comentário de nota de rodapé que Salmon fez ao introduzir o capítulo sobre os métodos das polares recíprocas na terceira edição, de 1855 , de $A$ treatise on conic sections, ao dizer, por exemplo, que o princípio da dualidade, o qual envolve o referido método, em $A$ treatise on higher plane curves, pode ser visto com um tratamento puramente analítico (SALMON, 1855 , p. 253).

A seguir estão alguns dos resultados que Salmon publicou na edição do seu segundo tratado, os quais ele já havia apresentado em A treatise on conic sections em uma abordagem sintética, mas depois com uma perspectiva analítica.

Os artigos 301 e 302 de A treatise on conic sections e os Artigo 239 e 240 de A treatise on higher plane curves refletem exatamente esta dualidade de abordagem: enquanto os dois primeiros artigos fazem um tratamento sintético sobre os métodos projetivos basendo nas propriedades das razões anarmônicas, os dois últimos fazem a abordagem desse método adotando as equações homogêneas para as retas em questão.

A razão anarmônica para o referido feixe de retas é dada por $\frac{(a-b)(c-d)}{(a-c)(b-d)}$ e no sistema de coordenadas trilineares, as retas são dadas por pelas três coordenadas, $\lambda-$ $\alpha \mu, \lambda-\beta \mu, \lambda-\gamma \mu, \lambda-\delta \mu$.

\section{CONSIDERAÇÕES FINAIS}

O desenvolvimento desse artigo permitiu primeiramente visualizar as transformações que ocorreram em um determinado período da história e que impactaram efetivamente na ordem social. Tal momento residiu no século XIX, o qual observou significativas mudanças que não só aconteceram no âmbito da matemática.

O século XIX registrou o impulso que a Revolução Industrial promoveu na sociedade, de modo que a influência dessa revolução atingiu obviamente a ciência. Desse modo, o reconhecimento do cenário de um determinado período em uma análise para realizar uma pesquisa foi fundamental para poder compreender os fatos que se seguiram, principalmente, no que diz respeito à produção dos novos resultados matemáticos naquele período. Um deles foi o desenvolvimento de álgebras (abstratas) e geometrias (não euclidianas) diferentes da álgebra e da geometria que havia até então, sobretudo, os resultados e as práticas inovantes da geometria projetiva.

Além disso, outro ponto necessário foi limitar o campo de pesquisa, o que permitiu focar em um local mais específico, que foi adotar a matemática britânica do século XIX como referência para a pesquisa. 
Ao centrar o momento e o espaço de estudo, levantaram-se as questões quanto às abordagens adotadas por Salmon. No primeiro momento, identificou-se que a matemática britânica estava muito fundamentada nas suas tradições sintéticas oriundas da herança deixada por Taylor, MacLaurin e Newton, preservando, dessa forma, seu tratamento sintético nos seus resultados, o que impactava tanto nas pesquisas quanto no ensino daquela época.

Esse dogmatismo fez com que a matemática britânica se colocasse em um estado de isolamento, distanciando-se cada vez mais dos desenvolvimentos da matemática dos demais países da Europa que já adotavam uma abordagem analítica em seus resultados.

Porém esse estado de isolamento começou a sofrer uma abertura através de movimentos liderados, inicialmente por Robert Woodhouse, o qual introduziu no início do século XIX na matemática britânica os primeiros resultados cujos tratamentos eram analíticos. Após Woodhouse, matemáticos britânicos, como George Peacock, formaram a chamada Analitycal Society que estabeleceu definitivamente a adoção também do tratamento analítico na matemática britânica.

Esses fatos transcorrem na primeira metade do século XIX, já na outra metade, com os tratamentos analíticos, as figuras que ascendem são Cayley, Sylvester e George Salmon, que firmam uma prática comum que foi a produção de resultados da álgebra aplicados à geometria, culminando no desenvolvimento da teoria dos invariantes e, sobretudo, na publicação dos quatro tratados de Salmon, os quais em suas edições apresentavam os recentes resultados da geometria projetiva daquele momento, como o princípio da dualidade e os métodos de projeção.

A publicação desses resultados faz parte de uma série de trabalhos que não só ocorreu na matemática britânica e que também ganhou uma posição de destaque na matemática do século XIX, tornando-se um campo muito ativo de pesquisa com vários matemáticos ilustres envolvidos nesse desenvolvimento.

A geometria projetiva serviu de palco para a rivalidade entre as abordagens sintética e analítica. Monge foi uma figura que atuou nas duas frentes, de modo que buscava harmonizar a relação entre esses dois campos. Tão logo a sua morte, levantaram-se para rivalizar as figuras de Poncelet e Gergonne, ambos discípulos de Monge, em que o primeiro era um defensor dos métodos sintéticos, enquanto o segundo era defensor dos métodos analíticos.

Dessa forma, a publicação dos trabalhos de George Salmon só se pode entender pelo contexto em que ele vivia e trabalhava, ou seja, no contexto da matemática britânica, sobretudo na Irlanda e na Inglaterra. Nesse contexto identificou-se que por volta de 1850 há uma grande virada na matemática britânica que se traduz, tanto no ensino quanto na transmissão dos métodos ensinados em geometria, por uma ênfase com os métodos analíticos.

Isso se manifestou claramente nas duas primeiras obras de Salmon. De toda forma, percebeu-se que os métodos sintéticos continuaram servindo de suporte aos desenvolvimentos analíticos e estes, por sua vez, cobriram as lacunas do outro método, como ocorreu no tratamento dos sistemas de coordenadas, ao poder localizar um ponto que se encontra no infinito. 
Nesse sentido os trabalhos de Salmon foram muitos representativos tanto no ensino e quanto na pesquisa da matemática britânica, naquele período, não sendo por acaso que esses dois tratados foram traduzidos para diversos idiomas, com destaque para as traduções feitas para o francês e para o alemão.

Uma idéia de como a geometria projetiva se encontra na atualidade é que ela está presente em vários domínios, uma delas é a computação gráfica que está usando a representação analítica de pontos e retas por coordenadas homogêneas e a representação matricial das transformações projetivas.

\section{Bibliografia}

ANDRADE, Plácido e BARROS, Abdênago. 2010. A. Introdução à geometria projetiva. Rio de Janeiro, SBM.

BALL, Walter William Rouse. 1889. A history of the study of mathematics at Cambridge. Cambridge, Cambridge University Press.

CAYLEY, Arthur. 1845a. Mémoire sur les courbes à double courbure et sur les surfaces développables. In Journal de mathématiques pures et appliquées, 1re série, tome 10, pp. 245-250.

CAYLEY, Arthur. 1845b. Démonstration d'un theorem de M.Chasles. In Journal de mathématiques pures et appliquées, 1re série, tome 10, pp. 383-384.

CAYLEY, Arthur. 1848. On geometrical reciprocity. In Cambridge and Dublin mathematical journal, vol. 3, pp. 173-79, reed. CAYLEY, A. The collected mathematical papers of Arthur Cayley. Cambridge, University Press, vol. 1, 1889. pp. 377-382.

CAYLEY, Arthur.1858b. A fifth Memoir upon Quantics. In Philosophical Transactions of Royal Society of London, reed. CAYLEY, A. The collected mathematical papers of Arthur Cayley. Cambridge, University Press, vol. 2, 1889. pp. 527-557.

CAYLEY, Arthur. 1859. A sixth Memoir upon Quantics. In Philosophical Transactions of Royal Society of London, reed. CAYLEY, A. The collected mathematical papers of Arthur Cayley. Cambridge: At The University Press, vol. 2, 1889. pp. 561-592.

CHASLES. Michel. 1837. Aperçu historique sur l'origine et le développement des méthodes en géométrie. Bruxelles, M. Hayez.

CHASLES. Michel. 1852. Traité de géometrie supérieure, par M. Chasles. Paris, Bachelier, Imprimeur-libraire de l'école polytechine.

CRILLY, Tony. 1986. The Rise of Cayley's invariant Theory (1841 - 1862). In Historia Mathematica, vol. 13, pp. 241-254.

DIAS, Leandro Silva e GRIMBERG, Gerard Emile. 2015. Álgebra e geometria projetiva analítica na Inglaterra dos anos 1841 - 1853. In Llull, Madrid, v. 38, pp. 11-31.

FISCH, Menachem. 1994. 'The emergency which has arrived': the problematic History of Nineteenth-Century British Algebra. In The British Journal for the History of Science, vol. 27, no. 3, pp. 247-246.

GOW, Rod. 1997. George Salmon 1819 - 1904: his mathematical work and influence. In Irish Math. Soc. Bull. no. 39. pp. 26-76.

GUICCIARDINI, Niccolò. 1989. The Development of Newtonian Calculus in Britain 1700 - 1800. Cambridge: Cambridge University Press. 
JOLY, Charles Jasper. 1905. George Salmon 1819 - 1904. Proc. Royal Society 75, pp. 347355.

LORENAT, Jemma. 2015. Figures real, imagined, and missing in Poncelet, Plücker, and Gergonne. In Historia Mathematica, Elsevier, pp. 155-192.

LORENAT, Jemma. 2016. Synthetic and analytic geometries in the publications of Jakob Steiner and Julius Plücker (1827-1829). In Archive for History of Exact Sciences, vol. 70, pp. 413-462.

MCCONNELL, Albert Joseph. 1970-1990. Biography of Salmon, in Dictionary of Scientific Biography. New York, Charles Scribner's Sons, vol.12.

MANSION, Paul. 1873. Notice sur les travaux de Jules Plücker. In Bulletin des sciences mathématiques et astronomiques, vol. 5, pp. 313-319.

MENGHINI, Marta. 2019. Luigi Cremona and Wilhelm Fieldler: the link between descriptive and projective geometry in technical instruction. In: Evelyne Barbin, Marta Menghini \& Klaus Volkert (org.), Descriptive Geometry - The Spread of a Polytechic Art and the Legacy of Gaspard Monge. Cham: Springer.

MOLLAN, Charles. 2007. It's Part of What We Are: some irish contributors to the development of the chemical and physical sciences (science and irish culture). vol. 2, Hardcover.

PARSHALL, Karen Hunger. 1989. Toward a history of nineteenth-century invariant theory. In: ROWE, D. E.; MCCLEARY, J. The history of modern mathematics. California, San Diego: Academic Press, vol. 1, pp. 157-206.

RICHARDS. Joan. 1986. Projective geometry and mathematica progress in mid-victorian britain. Stud. Hist. Phil. Sci., vol. 17, No. 3, pp. 297-325.

RICHARDS. Joan. 1992. God, Truth, and Mathematics in nineteen century England. The Invention of Physical Science Kluwer Academic Publishers, pp. 51-78.

ROSENFELD, Boris Abramovich. 1988. A History of Non-euclidean Geometry, Evolution of the Concept of a Geometric Space. New York: Springer-Verlag.

SALMON, George. 1846. On the degree of a surface reciprocal to a give one. In Cambridge and Dublin mathematical journal, vol. 2, pp. 65-75.

SALMON, George. 1848. On the condition that a plane should touch a surface along a curve line. In Cambridge and Dublin mathematical journal, vol. 3, pp. 44-46.

SALMON, George. 1848. On the numbers of normals which can be drawn from a given point to a given surface. In Cambridge and Dublin mathematical journal, vol. 3, 1848, pp. 46-47.

SALMON, George. 1852. Théorèmes sur les courbes de troisième degré. In J. für die reine und angewandte Math., pp. 274-276.

SALMON, George. 1850. A treatise on conic sections. $2^{\mathrm{a}} \mathrm{ed}$, London: Longman, Brown, Green, and Longmans. $3^{\mathrm{a}}$ ed. London: Longman, Brown, Green, and Longmans, 1855.

SALMON, George. 1851. Sur la formation de l'équation de la courbe reciproque à une courbe donnée. In Journal für die reine und angewandte Mathematik, vol. 42, pp. 277-278.

SALMON, George. 1852. A treatise on the higher plane curves. $1^{\mathrm{a}}$ ed., Longman, Brown, Green, and Longmans, London. $3^{\mathrm{a}}$ ed. London: Longman, Brown, Green, and Longmans, 1879. 
SALMON, George. 1859. Lessons introductory to the modern higher algebra, Hodge, Smith and Co, Dublin.

SALMON, George. 1862. A treatise on the analytic geometry of three dimensions, Hodge, Smith and Co, Dublin.

SYLVESTER, James Joseph. 1850. On the intersections, contacts, and other correlations of two conics expressed by indeterminate coordinates. In Cambridge and Dublin mathematical journal, vol. 5, pp. 262-285, reed. SYLVESTER, J. J. The collected mathematical papers of James Joseph Sylvester. Cambridge: At The University Press, Vol. 1, 1904. pp. 119-137.

SYLVESTER, James Joseph. 1851. On certain general properties of homogeneous functions. In Cambridge and Dublin mathematical journal, vol. 6, pp. 1-31, reed. SYLVESTER, J. J. The collected mathematical papers of James Joseph Sylvester. Cambridge: At The University Press, vol. 1, 1904. pp. 165-180.

SYLVESTER, James Joseph. 1851. On the general theory of associated algebraical forms. In Cambridge and Dublin mathematical journal, vol. 6, pp. 289-293, reed. SYLVESTER, J. J. The collected mathematical papers of James Joseph Sylvester. Cambridge: At The University Press, vol. 1, 1904. pp. 198-202.

VOLKERT, Klaus. 2019. Otto Wilhelm Fiedler and the synthesis of projective and descriptive geometry. In: Evelyne Barbin, Marta Menghini \& Klaus Volkert (org.), Descriptive Geometry - The Spread of a Polytechic Art and the Legacy of Gaspard Monge. Cham: Springer, pp. 168-181.

WILSON, Edwin Bidwell. 1903. The synthetic treatment of conics at ter present time. In Bull. Amer. Math. Soc.vol. 9, no 5, pp. 248-254.

Rodolpho Sousa Lima, Mestre PEMAT - UFRJ,
rodolphors1@ gmail.com
$\begin{aligned} & \text { Gérard Emile Grimberg, Pr. PEMAT - UFRJ } \\ & \text { gerard.emile@ @erra.com.br }\end{aligned}$

RBHM, Vol. 19, n 38, p. 45-81, 2019 